\title{
Enhancing the use of emergency contraception in a refugee setting: Findings from a baseline survey in Kakuma refugee camps, Kenya
}

Esther G. Muia

Joyce Olenja

Follow this and additional works at: https://knowledgecommons.popcouncil.org/departments_sbsr-rh

Part of the International Public Health Commons

How does access to this work benefit you? Let us know!

\section{Recommended Citation}

Muia, Esther G. and Joyce Olenja. 2000. "Enhancing the use of emergency contraception in a refugee setting: Findings from a baseline survey in Kakuma refugee camps, Kenya." Nairobi: Population Council. 
Enhancing the Use of Emergency Contraception in a Refugee Setting: Findings from a Baseline Survey in Kakuma Refugee

Camps, Kenya

Esther Muia*

Fariyal F. Fikree**

Joyce Olenja***

Population Council, Nairobi*

Population Council, New York**

Consultant, Population Council*** 


\section{TABLE OF CONTENTS}

ACKNOWLEDGMENTS i

LIST OF DATA TABLES ii

LIST OF ABBREVIATIONS iii

CHAPTER 1: $\quad$ Introduction $\quad 1$

CHAPTER 2: $\quad$ Methodology

Quantitative Component

Qualitative Component

CHAPTER 3: $\quad$ Results

Quantitative Component

Health Care Providers

Refugee Women

Qualitative Component

Focus Group Discussions

Adolescents: Male and Female

Women Support Groups

Community Male Elders

CHAPTER 4: $\quad$ Discussion

CHAPTER 5: $\quad$ Recommendation

Appendix $1 \quad$ Questionnaire - Health Care Providers

Appendix 2 Questionnaire-Refugee Women

Appendix 3 Focus Group Discussions - Guidelines 


\section{ACKNOWLEDGMENTS}

The baseline survey as a preliminary step to enhance the use of emergency contraception in a refugee setting was funded by Compton Foundation through the International Rescue Committee; without their generosity and continuous support the project could not have accomplished what it has to date.

We would like to thank all the individuals who provided assistance in development, conduct, analysis and report writing. We would also like to thank the interviewers, supervisors, editors and data entry operators and others too numerous to mention who assisted us admirably in various stages of this project.

Special appreciation goes to Dr. Stephen Redding, the Country Director for IRC who was instrumental in ensuring the start of the project, with support from Dr. James Gesami of the Ministry of Health, together with members of the Health and Nutrition Coordination Committee. Thank you to Dr. Robin, Dr. Elekima, Florence Ndung'u, Grace Muthumbi and all the staff of IRC in Nairobi and Kakuma as well as Mr. Baraza of NCCK in Kakuma for their continuos support in various ways. We appreciate the long hours that Eugenia King' ori and Jennifer Liku put into organising the data.

And, last but not least Dr. Ayo Ajayi, Regional Director of the East and Southern Africa office of Population Council Nairobi, for his continued advice and support. 


\section{LIST OF ABBREVIATIONS}

$\begin{array}{lll}\text { BPRM } & = & \text { Population, Refugees and Migration } \\ \text { CBD } & = & \text { Community Based Distributor } \\ \text { CBR } & = & \text { Community-Based Rehabilitation } \\ \text { CHW } & = & \text { Community Health Worker } \\ \text { EC } & = & \text { Emergency Contraceptive } \\ \text { EPI } & = & \text { Expanded Program on Immunization } \\ \text { FGDs } & = & \text { Focus Group Discussions } \\ \text { FP } & = & \text { Family Planning } \\ \text { HCPs } & = & \text { Health Care Providers } \\ \text { IEC } & = & \text { Information Education and Communication } \\ \text { IRC } & = & \text { International Rescue Committee } \\ \text { LTNHCR } & = & \\ \text { MISP } & = & \text { Minimal Initial Service Package } \\ \text { MVA } & = & \text { Manual Vacuum Aspiration } \\ \text { NCCK } & = & \text { National Christian Council of Kenya } \\ \text { OLS } & = & \text { Operations Lifeline Sudan } \\ \text { RH } & = & \text { Reproductive Health } \\ \text { STDs } & = & \text { Sexually Transmitted Diseases } \\ \text { SV } & = & \text { Dutch Refugee Foundation Stichting Vluchteling } \\ \text { TBA } & = & \text { Uraditional Birth Attendant } \\ \text { UNHCR } & = & \end{array}$




\section{INTRODUCTION}

The Kakuma Refugee Camp is located in the Turkana District of Kenya, 127 kilometers south of the Kenya-Sudan border and $120 \mathrm{Kms}$ northwest of Lodwar. In August 1992 Kakuma refugee camp was established to receive over 20,000 refugees fleeing insecurity in Southern Sudan. The camp population has expanded steadily since that time and now houses refugees from over 10 countries. Currently the camp population is 79,316 refugees, of whom approximately 38,000 are of reproductive age. The camp continues to grow steadily and many of the new arrivals from Southern Sudan are women and children. Children under the age of 5 account for $14 \%$ of the total population, while $40 \%$ of the refugees are women, although this percentage continues to increase.

Sudanese refugees make up approximately $71 \%$ of the total population while Somalis are the second largest population, comprising $24 \%$. The number of refugees from Ethiopia has now declined to only about $4 \%$. The remainder of the camp population is comprised of refugees from various nations, including Uganda, Rwanda, Burundi and the Democratic Republic of Congo.

There are approximately 150 births in the camp per month, 50 percent of which are TBA assisted in the community. Antenatal and family planning services are offered in three of the satellite International Rescue Committee (IRC) clinics.

\section{The Role of IRC}

In August 1992, IRC at the request of the United Nations "Operation Lifeline Sudan" (OLS), the United Nations High Commission for Refugees (UNHCR) and the Kenyan Government, initiated a primary health care program in the Kakuma Refugee Camp. The U.S. Bureau for Population, Refugees and Migration (BPRM), and the Dutch Refugee Foundation Stichting Vluchteling (SV) funded the program with co-funding from the Dutch Government and LTNHCR.

Since that time, the population of the camp has continued to grow, and activities have moved from a crisis to a maintenance phase. In January 1997, IRC assumed the additional responsibility of the camp hospital, bringing the entire health sector under the management of IRC. IRC's programs now focus on two areas: maintaining and improving public health and promoting self-reliance' particularly of the most vulnerable communities.

IRC's health services in Kakuma camp currently consist of:

- A 90 bed primary care hospital in the camp

- Five community-based clinics providing curative outpatient services, as well as preventative services including family planning services (at three of the clinics), ante- and 
post-natal care, an expanded program for immunization (EPI) and nutrition programs for the refugees and the local population.

- An extensive community outreach program focusing on preventative care and public education. The team includes Community Health Workers (CHWs), Traditional Birth Attendants (TBAs), EPI Vaccinators, Reproductive Health Field Workers and Mental Health Workers.

- A Community-Based Rehabilitation (CBR) Program, serving the special therapeutic and social needs of the handicapped and aged population of the camp and local Turkana population. This program has a well-developed training component, enabling the disabled to acquire skills and participate in production and income-generating activities.

All IRC's programs emphasize meeting the special requirements of vulnerable populations, specifically women, the disabled and adolescents. Initiatives are open to the host Turkana community as well as the refugees, and about $10 \%$ of current service beneficiaries are from the local area.

\section{Location}

Kakuma is located $127 \mathrm{~km}$ south of the Kenya/ Sudan border and $120 \mathrm{~km}$ northwest of the town of Lodwar in Turkana District. The camp itself is divided into three sections, Kakuma I and Kakuma II and Kakuma III. The main camp (Kakuma I) has a hospital, five clinics and over 25 schools, two distribution centers, a library, markets and functioning water and sanitation systems.

The extension areas, Kakuma II and III, are located $12 \mathrm{~km}$ to the west of Kakuma I, across the Lodoket River. Kakuma II covers an area of 20 square Kms. It was founded in November 1997 and is divided into 3 phases, populated primarily by members of the Banjuni, Bantu and Barawa Somali ethnic groups. With the recent arrival of large numbers of Southern Sudanese, an additional extension area, Kakuma III was opened in January 1999. The latest extension covers a smaller area, is in the process of constructing more durable housing, and is further west about $2 \mathrm{~km}$ away from Kakuma II. The predominant group here is Sudanese. A number of the refugees are still housed in temporary communal shelters where new arrivals disembark currently.

Services are more limited in Kakuma II and III as compared to the older, more established refugee camp in Kakuma I. Although IRC has recently opened a health clinic in Kakuma (ESHTER PLEASE MENTION HERE WHETHER IN KAKUMA II OR III), the refugees must still travel to Kakuma I for some curative health services, and to participate in most community based rehabilitation projects.

The terrain is extremely arid, with rainfall averaging $350 \mathrm{~mm}$ per year. Rainfall is seasonal, coming typically between April and November, and often causing flooding. Shortage of water, and the limited land surface to which the refugees have access, continue to hinder the ability of 
refugees to produce enough food to become self-sufficient. In addition, the local Turkana population is resistant to refugees establishing livestock industries, and has been known to raid the camp to steal goats.

\section{Beneficiaries}

The project will focus on emergency contraception, as an aspect of reproductive health need of refugee women and men of reproductive age in Kakuma as has been identified in an earlier reproductive health needs assessment study. The population of women of reproductive age (1544 ) is currently 13,144 and the population of men of reproductive age (15-59) is 25,763, bringing the total beneficiary target population to $38,907^{4}$. The program will especially target the large adolescent community currently resident in the camp, which makes up about $40 \%$ of the camp population.

\section{Problems to be addressed}

The two factors that influenced the evolution of this project were:

1. The Interagency Working Group on Reproductive Health for Refugees recommendations for a Minimal Initial Service Package (MISP) of reproductive health services during the emergency phase of a refugee crisis. The MISP contents include prevention and the management of the consequences of sexual and gender violence including the provision of emergency contraception (EC).

Emergency contraception, relegated to the shadows until recently, is relatively unknown in the general population in Kenya. Yet the method has great potential, since oral contraceptives are relatively easily available in the country. Established methods include the Yuzpe regimen, the levonorgestrel regimen and the copper IUCD. The Yuzpe regimen, which is combined oral contraceptive pills taken four to eight times the daily dose for one day and within 72 hours of unprotected sexual intercourse, has been popular. More recently the levonorgestrel regimen have been created using the ordinary progestin only pill, and has been widely used most recently in a larger national programme that enhanced the use of EC in the general population in Kenya. The dedicated product used then is the Postinor pill, which are packaged either in packets of 10 or two tablets.

Furthermore, the Interagency Field Manual for Reproductive Health in Refugee Situations statement that "sexual and gender-based violence is strongly associated with situations of forced population movement" and "In this context it is vital that emergency post-coital contraception supplies are available to those who request this." strengthens the argument for the provision of emergency contraceptives in the Kakuma refugee camps. 
2. Findings from a comprehensive Reproductive Health Survey and Facilities Review completed in Kakuma in October 1998 by IRC confirmed that knowledge of reproductive health issues in the camp was low, and that further intervention was essential to increase knowledge and adoption rates of positive behaviors regarding a range of reproductive health issues, including EC, where knowledge on this specific method was noted to be particularly low.

\section{The Reproductive Health Survey}

The survey questioned 686 women and 605 men of reproductive age on knowledge, attitudes and behaviour (KAB) related to family planning, safe motherhood, emergency obstetric care, sexual and gender-based violence and STDs/HIV. The sociodemographic profiles of men and women surveyed closely resemble that of the camp population.

Although IRC and National Council of Churches of Kenya (NCCK) are providing education on RH topics in Kakuma, preliminary survey results indicate that education needs to be intensified. For example, the survey showed that only $35 \%$ of women in the camp were able to cite any modem methods of contraceptives, and only $36 \%$ knew that contraceptives were available at camp health facilities. Current use of modern contraceptives among women was also only $8 \%$.

The survey and facility review highlighted the need to improve knowledge about and access to emergency reproductive health services in the camp, including emergency obstetric and emergency contraception. Currently EC is only available at the camp hospital. In addition, of the $34 \%$ of women and $27 \%$ of men who stated that women are forced to have sex in the camp, only $11 \%$ knew that emergency contraception was available through the health service system.

Gender violence emerged as a significant problem within the camp, both with regards to rape and domestic violence. Not only were reports of forced sex high, but also certain forms of gender violence maintained high levels of acceptability. For example, $12 \%$ of women reported having been beaten by a spouse or partner in the previous month, a figure that is likely to represent the lower bound of true incidence. Of the total population surveyed $40 \%$ of women and $39 \%$ of men indicated that it was appropriate for men to beat their wives. Among Sudanese respondents (the major ethnic group of the camp) $70 \%$ of women and $87 \%$ of men indicated that the behavior is appropriate.

It was recommended that training was needed for senior staff on quality assurance including management and logistics, monitoring and proper collection and analysis of data. Recommendations for clinical staff included training on essential drugs and clinical diagnosis, postpartum and post-abortion care, FP and STD counseling skills, emergency contraception and ethics and privacy. 


\section{Project Objectives:}

\section{$\underline{\text { Overall }}$}

To contribute to the improved quality of reproductive health services for refugees and the local Turkana population in Kakuma through operations research on the introduction of emergency contraception.

\section{Specifically}

1. To expand family planning access to women who require them

2. To enhance the use of emergency contraception in a refugee setting

3. To link the expertise of two organizations, a research organization and a service delivery organization, in improving the quality of reproductive health care delivery

4. To document and share the lessons learned in order to adapt the use of emergency contraception in a refugee setting.

The current report will present the findings from the quantitative as well as the qualitative aspects of the baseline survey. This preliminary report will be shared with the community from the camps, including providers and policy makers. It is anticipated that this information will assist in the revision of the training curricula for the health care providers in the refugee camps, and the development and mode of dissemination of IEC messages in the community and health facilities. 


\section{METHODOLOGY}

\section{A. QUANTITATIVE COMPONENT}

Two quantitative survey instruments focusing on reproductive health in general and Emergency Contraception (EC) in particular were designed to explore knowledge, use and attitude regarding emergency contraceptives among women (target sample size of approximately 1,000 women of reproductive age residing in Kakuma camps I, II and III); and health care providers (target sample size of approximately 30 health care providers based in clinics and the camp hospital in Kakuma camps I, II and III).

Interviewers who included staff from IRC and refugee Community Health Workers (male and female) were trained for two days by staff from the Population Council. The data collection was supervised by Council staff in collaboration with one of the doctors of IRC, who was interested in gender issues including rape. Data were collected in all the 3 camps (Kakuma I, II and III). There were no refusals among the refugee respondents.

\section{Survey among Health Care Providers}

Instruments developed earlier for a similar survey conducted in Kenya on emergency contraception were modified for this survey bearing in mind the target population for the proposed project. They were reviewed by peers both in Nairobi and New York offices of IRC and Population Council. Pre-testing was conducted in Kakuma I in a zone that was not to be included in the baseline survey later. Because professional providers are so few in the Kakuma refugee camp, all health providers present in the camp at the time of the survey were included in the survey. There were no refusals. Among the community health providers, all those found at the facility on the day of the interview were interviewed.

As reflected in the instruments (see Appendix 1) information was sought on:

- Nationality

- Socio-demographic characteristics

- Professional qualifications including work experience

- Reproductive health services provided

- Family planning clients served

- In-service family planning training

- Opinion on who should be provided with emergency contraceptives

- Service delivery practices for emergency contraceptives

- Knowledge and attitude to emergency contraception

\section{$\underline{\text { Survey among Refugee Women }}$}


Data collection instruments previously used by Kenyan colleagues in similar research were modified/revised (see Appendix 2) following consultations with peers in Kenya and New York. The following information was sought:

- Socio-demographic characteristics

- Pregnancy and family planning practices, including method use

- Knowledge and attitude regarding emergency contraception

- Concerns regarding emergency contraception

- Availability of the method in the refugee camp setting

In each camp, that is, Kakuma I, II and III a list of women aged 15 years above was drawn with assistance from the elders and the IRC office at the camp. From these a proportionate allocation of women was randomly sampled, and their houses identified with the assistance of guides who were from the specific camps. Women who were community health providers were excluded from the sampling strategy. After obtaining informed consent verbally, women were interviewed at their residence. Those not found at home at the time of the interview were followed up the next day with appointment. Due to security reasons, there was no data collection after six in the evening and the interviewers returned to their base at that time.

\section{Survey of Policy-makers}

There were difficulties in accessing some of the policy makers at the camp. Only two policy makers were interviewed, this was due to their availability at the time. As a result we will not present these results independently, as they will not be a representative sample of the policy makers of the community. However, we managed to conduct a focus group discussion with the elders from the various communities at the camp and will present this in the relevant section of the report.

\section{$\underline{\text { Data Entry for Quantitative Survey }}$}

Data was edited and cleaned while in the field and open-ended questions coded at Population Council/Nairobi, where data entry and analysis were conducted. The findings are reported in the following chapter.

\section{B. QUALITATIVE COMPONENT}


The quantitative data was complimented by a qualitative component using focus group discussions [FGDs]. In total eight focus group discussions were conducted, each comprising between five and twelve participants. Information was sought on (see Appendix 3):

- Knowledge on Contraception including EC

- Reasons for Using EC

- Concerns on EC usage

- Views and attitudes regarding content, clientele and mode of dissemination of EC information

- Distribution points for ECs

The focus group discussions were conducted with opinion leaders, adolescents (male and female) and representatives from the women support group in the Kakuma Refugee camp.

\section{Conduct of the Focus Group Discussions}

The composition of the eight focus group discussions were as follows:

\section{Adolescents aged between 14 and 21 years 6 FGDs were conducted with:}

Somali female aged between

Sudanese female aged between

Sudanese mixed male and female adolescents

Sudanese male

Somali male

Mixed ethnic male

\section{Opinion leaders [2 FGDs]}

Somali men

Sudanese men

\section{Women Support Group [1 FGD]}

This consisted of women from the Sudanese, Somali and Uganda ethnic groups

All participants were identified through each ethnic group's social network in the group, and the participants had to be able to speak and understand basic English as this was the medium of communication.

At the beginning of each FGD, the moderator started off the discussion by introducing herself and the note taker as researchers from The Population Council in Nairobi working in the field of health, reproductive health in particular. Having set the pace she then requested each of the participants to introduce themselves by stating their names and ages. The age range for the adolescent FGDs irrespective of gender were between 14 to 22 years.

The moderator then introduced the subject of discussion within the broader context of reproductive health. Because of the sensitivity of the subject, the approach varied depending on 
the initial mood of the group. On the whole it was much easier to introduce the subject among the youth who displayed curiosity from the on-set. Their responsiveness may also be attributed to the fact that issues of adolescent health including sex, pregnancy and STDs were more pertinent to them and often they had no answers to most of their concerns on these issues. A forum for open discussion was therefore most welcome. This was evident in their active participation during the discussions and raising various questions that appeared to have bothered them for some time.

On the other hand, the men were often on the defensive to begin with, sensing it as an assault to their culture, which they considered already endangered by their refugee status. They were therefore keen to preserve as much of their culture as possible. However, an assurance to the effect that their culture, like any other, is respectable and the fact that the subject for discussion would take into account the cultural context, paved the way for an open discussion that was often fruitful and revealing. Across the board, irrespective of age or ethnicity, males posed as the custodians of culture which could have resulted in unsuccessful discussions. However, the moderator clarified issues and concerns regarding emergency contraceptives from the onset and drew heavily upon the participants' culture, as a referent. This was a key strategy for steering the discussion forward in any meaningful way.

\section{Data Entry for Qualitative Survey}

The notes from the FGDs were transcribed and a detailed analysis conducted. The results of the discussions follow the quantitative findings. 


\section{FINDINGS}

\section{A. QUANTITATIVE COMPONENT}

\section{i) Health Care providers [HCPs]}

A total of 16 health care providers [HCPs] of whom 12 were female were interviewed for the baseline survey. The majority (87.5\%) of the HCPs were either Kenyans (56.3\%) or Sudanese $(31.3 \%)$ and mainly served the clientele located in the vicinity of Clinics 1,5 and 6 serving the refugee community in Camp Kakuma I. Only one clinic was located in Kakuma II whilst none of the HCPs directly serving the population of Kakuma III were interviewed. The youngest HCP was 25 years of age whilst the eldest was 41 though nearly $43 \%$ of the respondents were aged 35 and over.

Table 1: $\quad$ Percentage of health care providers $(n=16)$ interviewed with selected Background characteristics

\begin{tabular}{|l|cc|}
\hline \multicolumn{1}{|c|}{ Characteristics } & \multicolumn{2}{c|}{ Health Care Providers } \\
& $\mathrm{n}$ & \\
\hline Country of Origin & 9 & 56.3 \\
Kenya & 5 & 31.3 \\
Sudan & 1 & 6.3 \\
Burundi & 1 & 6.3 \\
Congo & & \\
\hline Sex & 12 & 75.0 \\
Female & 4 & 25.0 \\
Male & & \\
\hline Facility Location & 3 & 18.8 \\
Clinic 1 & 2 & 12.5 \\
Clinic 2 & 1 & 6.3 \\
Clinic 3 & 2 & 12.5 \\
Clinic 4 & 3 & 18.8 \\
Clinic 5 & 5 & 31.3 \\
Clinic 6 & & \\
\hline Camp Site Location & 15 & 93.7 \\
Kakuma I & 1 & 6.7 \\
Kakuma II & \multicolumn{2}{|}{} \\
\hline Current Age & 5 & 35.7 \\
25 - 29 & 3 & 21.4 \\
30 - 34 & 6 & 42.9 \\
35+ & \multicolumn{2}{c|}{} \\
\hline
\end{tabular}

$1=$ Camp hospital 
$2=\mathrm{n}=14 ;$ No information on current age for two health care providers

Among the 16 HCPs interviewed there was only one doctor, a Kenyan male with ten years of experience, working at the camp hospital in Kakuma I for the past 6 months. Among the four Kenyan nurses interviewed, three were female and reported work experience ranging from seven to thirteen years. Of the five clinical officers interviewed, one was from Burundi. Interestingly, five of the six female family planning service providers were Sudanese though only three of them had received NCCK training for 15 days. In addition, most of them [4/6] had only started working recently - in the past three months. However, most of the sixteen respondents reported being family planning providers $(37.5 \%)$, of whom ten reported having received specialized family planning training either through the NCCK or from other sources. Majority (43.7\%) of the HCPS had ten or more years of experience with the least being two years and the longest serving HCP had worked for 15 years.

Table 2: $\quad$ Percentage distributions of professional characteristics of health care providers $(\mathrm{n}=16)$

\begin{tabular}{|l|cc|}
\hline Professional Characteristics & \multicolumn{2}{|c|}{ Health Care Providers } \\
& $\mathrm{n}$ & \\
\hline Current Job & & 6.3 \\
Doctor & 1 & 25.0 \\
Nurse & 4 & 31.3 \\
Clinical Officer & 5 & 37.5 \\
Family Planning Service Provider & 6 & \\
\hline \# of Years of Experience & & 31.3 \\
$<5$ & 5 & 25.0 \\
$5-9$ & 4 & 43.7 \\
$10+$ & 7 & 62.6 \\
\hline Duration of Work in Kakuma & & 18.7 \\
$<5$ & 10 & 18.7 \\
$5-9$ & 3 & 3 \\
$10+$ & 3 & \\
\hline
\end{tabular}

The camp hospital where the doctor and the four nurses provided care was open for services round the clock every day of the week. The clinical officers and family planning service providers catered to clients for six and a half to eight hours during the week and for four and half to five and a half hours on Saturdays but did not provide services on Sundays.

The average weekly numbers of clients seen by the 16 HCPs were reported to range from $600-$ 800 whereas only two HCP reported seeing up to 100 weekly patients for any services offered. Most of the clients visiting the facilities were Sudanese $(81.3 \%)$. Though the average age of 
clients served by the facility were, in the opinion of the HCPs, relatively young (56.3\%) but the age range of the clients attending these facilities were identified to either vary somewhat $(43.8 \%)$ or not vary much $(31.3 \%)$. [See Table 3 below]

\section{Table 3: $\quad$ Percentage distributions of demographic characteristics of clients served}

\begin{tabular}{|l|rc|}
\hline Characteristics & $\mathrm{n}$ & $\%$ \\
\hline Average Age & & \\
Young & 9 & 56.3 \\
Medium & 7 & 43.8 \\
\hline Age range of clients & 4 & 25.0 \\
Varies a lot & 7 & 43.8 \\
Varies somewhat & 5 & 31.3 \\
Does not vary much & & \\
\hline Ethnicity & 13 & 81.3 \\
Sudanese & 2 & 12.5 \\
Somali & 1 & 6.3 \\
Others & & \\
\# of Clients seen weekly & 2 & 12.5 \\
$50-100$ & 4 & 25.0 \\
$200-500$ & 10 & 62.5 \\
$600-800$ &
\end{tabular}

Family Planning Services

The family planning services currently offered by any of the HCPs for the catchment population residing in Kakuma refugee camps I and II included contraceptive pills (combined, minipill and emergency contraceptive [EC]) and condoms. Surgical procedures of tubal ligation and vasectomy were not offered by any of the HCPs. The average number of clients given information and supplies were highest for pills and there were no clients who were provided information for vasectomy or tubal ligation, most probably due to the fact that these services were not offered at any of the health care facilities located at the refugee camps. However, information and/or supplies were provided to clients, on average, two clients per month, for ECs though the doctor and nurse provided these to only those clients who visited the camp hospital.

The contraceptive supplies were mainly obtained from the camp hospital $(25 \%)^{\mathrm{a}}$ or Ministry of Health $(25 \%)$ whereas a little over $30 \%$ were obtained from various other sources. The contraceptive brands reported normally stocked at the clinics were Microgynon (73.3\%), Nordette (73.3\%), Microlut (33.3\%), Neogynon (13.3\%) and Microval (6.7\%). Of these, only Neogynon was not reported to be currently in stock while among the others Microgynon $(81.8 \%)$ and Nordette $(81.8 \%)$ were reported to be currently in stock most often. However

\footnotetext{
${ }^{\text {a }}$ Camp Hospital is located in Kakuma I
} 
seven HCPs reported that they were not sure of the type of brands that were currently in stock these included mainly those who reported normally stocking Microgynon and Nordette brands. Nevertheless, most of the respondents (86.7\%) reported that they had experienced no difficulties in stocking contraceptive pills.

Reproductive Health Services Offered [Table 4]

Information on reproductive health services offered other than family planning covered pregnancy and STDs screening. Pregnancy testing and termination were reported to be offered by five of the $16 \mathrm{HCPs}$ interviewed. The method of pregnancy termination offered that was generally MVA conducted at Kakuma I camp hospital.

Regular screening of STDs was reported by only 43.8 of the HCPs interviewed though most of them $(93.8 \%)$ reported that they offer treatment for STDs

Table 4: $\quad$ Percentage distributions of reproductive health services offered

\begin{tabular}{|l|cc|}
\hline Reproductive Health Services & $\mathrm{n}$ & $\%$ \\
\hline Pregnancy Testing & & \\
Yes & 5 & 31.3 \\
No & 11 & 68.8 \\
\hline Pregnancy Termination & & \\
Yes & 5 & 31.3 \\
No & 11 & 68.8 \\
\hline STD Screening & & \\
Yes & 7 & 43.8 \\
No & 9 & 56.3 \\
\hline STD Treatment & & \\
Yes & 15 & 93.8 \\
No & 1 & 6.3 \\
\hline
\end{tabular}

Knowledge and Attitude regarding Emergency Contraceptives

Despite EC and supplies being provided only to those clients who visited the camp hospital, thirteen of the 16 HCPs interviewed reported that they were aware of ECs. The various reasons why ECs are used varied from prevention of pregnancy $(46.2 \%)$, good overall $(30.8 \%)$ or good for rape victims $(30.8 \%)$. It is important to note that the HCPs who reported that ECs were either good overall or good for rape victims were not the same though there was overlap with at least two of the HCPs who thought that ECs help prevent pregnancy. Three of the 13 HCPs 
had the impression that ECs were either against religion/culture $(7.7 \%)$ or not good due to being an abortifacient (15.4\%). Most of the HCPs $(61.5 \%)$ had heard about ECs in the recent past i.e. within the past two years and had heard about ECs either in the refugee camps at Kakuma or at the camp hospital.

Table 5: $\quad$ Percentage distributions regarding knowledge and attitude to Emergency Contraceptive

\begin{tabular}{|l|rc|}
\hline \multicolumn{1}{|c|}{ Emergency Contraceptive [EC] } & \multicolumn{2}{|c|}{ Health Care Providers } \\
Heard about EC & $\mathrm{n}$ & \\
Yes & 13 & 81.3 \\
No & 3 & 18.8 \\
\hline Impression regarding EC & \\
Helps prevent pregnancy & & 46.2 \\
Good overall & 6 & 30.8 \\
Good for rape victims & 4 & 30.8 \\
Should be given in an emergency & 4 & 7.7 \\
Not good as is an abortion & 1 & 15.4 \\
Against my religion/culture & 2 & 7.7 \\
Others & 1 & 15.4 \\
\hline Where heard about EC & 2 & 23.1 \\
College & & 30.8 \\
Kakuma Camp & 3 & 30.8 \\
Kakuma Camp Hospital & 4 & 15.4 \\
Others & 4 & 2 \\
\hline
\end{tabular}

$1=$ Multiple responses possible

The discussion with the HCPs on method of action, content and use of ECs raised several pertinent questions, which we suggest may assist in the development of IEC messages and training materials. The questions included:

1. What do you mean by unprotected sex?

2. Will EC prevent pregnancy if a woman has been raped?

3. Where can one get the method?

4. Can one get the method in the shops?

5. Are you expecting good results when you introduce this method?

6. Have you talked to people outside Kakuma about this method? If so how did they react?

7. What is the dosage?

The questions were asked either by the clinical officer or the family planning service provider except for the dosage question, which was asked by a nurse. 
Eleven of the 16 HCPS $^{b}$ interviewed reported that they did not offer ECs, as either they had not been instructed; non-provision of supplies or currently their facility was under repair. The latter was reported by seven of the $\mathrm{HCPs}^{\mathrm{c}}$ who were in the health center that was yet to be operational in Kakuma III.

\section{Service Delivery Practices for Emergency Contraceptive}

Among the five $\mathrm{HCPs}^{\mathrm{d}}$ who reported that they offer ECs, the reason for providing this service in the recent past was due to the increase in rape cases in the refugee camps. Only one provider mentioned that the provision of such services at the camp hospital followed a preventive program regarding Sexual and Gender Violence. However, ECs were provided, on average, once or twice a month to all clients without any selection criteria. The clients were provided ECs when they mentioned rape or unprotected sex irrespective of age or marital status.

There was no consistency regarding the EC regimen offered- the type, dosage and timing all varied among the five HCPs who provided this service though all worked at the camp hospital located at Kakuma I, which is where all rape cases are referred.

Counseling was offered to all clients who were provided ECs. The counseling covered such issues as appropriateness of use, method side effects and family planning counseling. There was no charge for providing ECs to clients. However, there were no IEC materials on EC or written standard service delivery guidelines for provision of EC available at the camp hospital.

The major obstacles regarding the utilization of ECs were low acceptability and hence underutilization of services and the impression among the clientele that ECs are a form of abortion. Interestingly, none of the HCPs mentioned that rape cases are not being reported hence rape victims do not benefit from the method or that some communities do not take the method even when prescribed suggesting that reasons for low acceptability did not pertain to reporting of rape cases ${ }^{\mathrm{e}}$ or non-compliance. We suggest that discussions with women and men in the communities be held to understand the reasons for low acceptability as a conduit for development of IEC materials and health education.

\section{Perceptions regarding Emergency Contraceptive}

Half of the HCPs interviewed perceived that EC was "good" while some clarified this by stating that EC was useful for rape victims (6.3\%) and that it prevented pregnancy (25\%). However, the remaining 50\% of HCPs mentioned either a lack of understanding of how "it prevents conception", an abortifacient, abuse of EC or is an emergency measure only. Despite the range in perceptions regarding EC, 11 of the 16 HCPs reported that EC is a suitable method due to the large number of rape cases in the refugee camps. However, only $25 \%$ of HCPs perceived "useful for rape cases" as an advantage of EC though "helps prevent pregnancy" was

\footnotetext{
${ }^{\mathrm{b}}$ Either clinical officers or family planning service providers

${ }^{c}$ Four clinical officers and three family planning providers

${ }^{\mathrm{d}}$ The doctor and four nurses

${ }^{\mathrm{e}}$ Paramount reason suggested by the HCPs for offering ECs
} 
perceived as an advantage by $50 \%$ of the HCPS interviewed. Other advantages perceived included minor side effects, women will benefit or offers a degree of protection though "does not protect from contracting sexually transmitted diseases" or is only $75 \%$ effective was also reported. Though most of the HCPs (31.3\%) reported no limitations to the use of EC, but high failure rate (12.5\%); and maybe abuse, limited duration of use following unprotected sex, unavailability, or cannot be used as a regular method among others were some of the perceived limitations reported.

A series of questions were asked regarding EC distribution as a method for emergency contraception in Kenya. Probing into the HCPs perspective for the reasons whether EC could be considered as a useful method for Kenya, protection against unplanned pregnancy (25\%) and reducing the rate of induced abortion (25\%) were the most common responses though women will benefit, good for young/unmarried women or for use in those communities where contraceptive use is low were also reported. However, a few HCPS were concerned about the abuse of EC and recommended that it be offered only to married women. ECs were suggested to be offered by medical personnel (87.5\%) though trained CHWs/TBAs were also recommended. Nurses or family planning service providers suggested recommendations for distribution of ECs by CHWs or TBAs but not by the clinical officers or doctor. However, all categories of health personnel interviewed recommended that medical personnel be considered the ideal candidate to offer this type of contraception. The clientele suggested for this method were rape victims $(12.5 \%)$, married or older women $(18.8 \%$ and $18.8 \%$ respectively) though $25 \%$ mentioned anyone who requests without contraindications. Further clarification listed several concerns including not being offered to adolescents, being offered to only those who are above 14 years of age and present with a complaint of unprotected sex or those who are in stable relationships but wish to delay their pregnancy. Though all 16 HCPs agreed to EC becoming more easily available in Kenya but voiced concerns regarding its advance distribution among clientele. Advance distribution of EC was either recommended as good $(50 \%)$ or good if provided only to women who are informed and/or literate $(31.3 \%)$. However, seven HCPs advised that advance distribution was either not good (12.5\%) or would lead to abuse $(31.3 \%)$. The most frequently reported mode for dissemination of information to women regarding EC was health facilities $(56.3 \%)$ or home visits $(50 \%)$ though other suggestions included women support groups $(18.8 \%)$, women meetings $(18.8 \%)$ and through TBAs $(12.5 \%)$.

The HCPs were next probed on their perceptions regarding advance distribution of EC within the refugee camps. Though $43.8 \%$ of the 16 HCPs interviewed felt that it would be a suitable idea for the refugee camps, but $31.3 \%$ were skeptical and two HCPs mentioned that either men would oppose its use or that it would be abused. Married (18.8\%) or literate women who understood the instructions $(25 \%)$ were generally recommended to receive advance distribution of EC but most of the HCPs $(75 \%)$ felt that they prefer EC to be more easily available to them.

Perceived challenges to provision of ECs were cultural $(18.8 \%)$ or religious $(12.5 \%)$ beliefs in contraceptive use and opposition by men (25\%) though three HCPs, all family planning service providers, reported that they did not foresee any challenge in providing ECs. Creating awareness about the advantages of ECs (76.9\%) was the most common suggestion for overcoming any obstacle in providing ECs. 
Most of the HCPs felt that special packaging of the ECs would facilitate its use though one $\mathrm{HCP}$, a nurse, pointed out that special packaging would not assist the illiterate women attending the clinics.

\section{ii) Refugee Women}

The majority of women were interviewed in the refugee camp located in Kakuma I that is the largest refugee camp in the Kakuma region. Most of the respondents interviewed (82.5\%) were either refugees from Sudan (50.5\%) or Somalia (32.0\%) with the remaining being either from Uganda (5.6\%) and Ethiopia (3.4\%) among others. The Sudanese respondents were mainly settled in Kakuma I (70.9\%) though some were from Kakuma III (28.9\%) whilst the Somali respondents were from either Kakuma I (36.1\%) or Kakuma II (63.5\%). Most of the respondents were in their twenties $(40.7 \%)$, the youngest was 11 and the eldest interviewed respondent was 65 years. Education as assessed by number of years of schooling was relatively high among the refugee respondents. Though $33.6 \%$ reported that they had never been to school (majority (51.1\%) of this group were from Sudan but an even larger proportion (36.0\%) reported that they had received more than 6 years of education (Sudanese comprising nearly $40 \%$ of this group too). [See Table 1]

Table 1: $\quad$ Percentage distribution of women $(\mathrm{n}=927)$ interviewed with selected background characteristics 


\begin{tabular}{|l|rr|}
\hline \multicolumn{1}{|c|}{ Characteristics } & $\mathrm{n}$ & $\%$ \\
\hline Refugee Camp & & \\
Kakuma I & 596 & 64.3 \\
Kakuma II & 191 & 20.6 \\
Kakuma III & 140 & 15.1 \\
\hline Country of Origin $^{1}$ & & \\
Sudan & 467 & 50.5 \\
Somalia & 296 & 32.0 \\
Uganda & 52 & 5.6 \\
Others & 109 & 11.8 \\
\hline${\text { Current } \text { Age }^{3}}_{10-19}$ & 250 & \\
$20-29$ & 375 & 27.1 \\
$30-39$ & 229 & 40.7 \\
$40-49$ & 63 & 24.8 \\
$50+$ & 5 & 6.8 \\
\# of Years of Schooling & 0.6 \\
None & & \\
$1-5$ & 307 & 33.6 \\
$6-12$ & 260 & 28.5 \\
$12+$ & 329 & 36.0 \\
\hline
\end{tabular}

$1=$ Missing information on 11 respondents

2 = Others include Congo $(\mathrm{n}=29)$, Burundi $(\mathrm{n}=24)$, Ethiopia $(\mathrm{n}=31)$, Rwanda $(\mathrm{n}=17)$ and Kenya $(\mathrm{n}=8)$

$3=$ Missing information on 5 respondents

$4=$ Missing information on 14 respondents

Over $70 \%$ reported that they had experienced at least one pregnancy; $23.4 \%$ were currently using any contraceptive method. Of these over $80 \%$ lived in Kakuma I and were mainly Somali refugees (38.7\%). Among the methods currently being used, pills $(52.1 \%)$ and Injectables (20.3\%) were the favored methods. Most Sudanese (63.5\%) reported pills as their current contraceptive method though Somali respondents mentioned pills (32.1\%) and condoms $(14.3 \%)$ as their favored current method. [Table 2].

Table 2: $\quad$ Percentage distribution of women $(\mathrm{n}=927)$ regarding pregnancy and family planning 


\begin{tabular}{|l|rc|}
\hline \multicolumn{1}{|c|}{ Characteristics $^{1}$} & $\mathrm{n}$ & $\%$ \\
\hline History of Pregnancy $^{1}$ & & \\
Yes & 686 & 74.4 \\
No & 236 & 25.6 \\
\hline Use a Contraceptive Method $^{\text {Yes }}$ & 217 & \\
No & 710 & 23.4 \\
\hline Method Use ${ }^{2}$ & & 76.6 \\
Pills & 113 & 52.1 \\
Injectables & 44 & 20.3 \\
Norplant & 7 & 3.2 \\
Condoms & 14 & 6.5 \\
IUCD & 12 & 5.5 \\
Withdrawal & 10 & 4.6 \\
Tubal ligation & 3 & 1.4 \\
Vasectomy & 1 & 0.5 \\
Others $^{3}$ & 13 & 6.0 \\
\hline
\end{tabular}

$1=$ Missing information on 5 respondents

$2=\mathrm{n}=217$

$3=$ Others include rhythm, currently breastfeeding and traditional

\section{Knowledge regarding Emergency Contraceptives}

The respondents $(\mathrm{n}=927)$ were questioned regarding their knowledge of options for women to consider following unprotected intercourse. Eleven percent (102 women) did not respond to this question. Of those who responded ( $\mathrm{n}=825$ ), nearly $56 \%$ were unaware that anything could be done to prevent a potential pregnancy following unprotected sex. The most frequently reported options were to go to the hospital $(25.7 \%)$, or use emergency contraceptives $(7.3 \%)$. However, $2.5 \%$ of the women reported that abortion could be considered following pregnancy confirmation. The likely options reported for women who went to the hospital $(\mathrm{n}=168)$ were for treatment $(31.5 \%)$, check-up $(29.2 \%)$ or prevent pregnancy $(17.8 \%)$. In addition, ten women reported provision of contraceptives such as pills, DPMA or condoms whilst eleven women recommended traditional methods. ${ }^{\mathrm{f}}$. [See Table 3 below]

Table 3: $\quad$ Percentage distributions for knowledge regarding emergency contraception

\footnotetext{
${ }^{\mathrm{f}}$ Withdrawal, hot water, salt and matchbox among others
} 


\begin{tabular}{|c|c|c|}
\hline Knowledge & $\mathrm{n}$ & $\%$ \\
\hline \multicolumn{3}{|l|}{ Avoid Pregnancy after unprotected sex } \\
\hline Nothing can be done & 458 & 55.5 \\
\hline Go to hospital or clinic & 212 & 25.7 \\
\hline Use traditional remedies & 11 & 1.3 \\
\hline Confirm pregnancy follow by abortion & 21 & 2.5 \\
\hline Use emergency contraception & 60 & 7.3 \\
\hline Others & 10 & 1.2 \\
\hline Not sure & 53 & 6.4 \\
\hline \multicolumn{3}{|l|}{ Ever heard about $\mathrm{EC}^{1}$} \\
\hline Yes & 137 & 14.8 \\
\hline No & 789 & 85.2 \\
\hline \multicolumn{3}{|l|}{ When heard about $\mathrm{EC}^{2}$} \\
\hline Past five years & 64 & 48.5 \\
\hline More than five years & 68 & 51.5 \\
\hline \multicolumn{3}{|l|}{ Where ever heard about $\mathrm{EC}^{3}$} \\
\hline Clinic & 82 & 59.9 \\
\hline Friends, family & 42 & 30.7 \\
\hline Read in newspapers, magazines & 16 & 11.7 \\
\hline Others $^{4}$ & 35 & 25.5 \\
\hline \multicolumn{3}{|l|}{ Where obtain EC EC $^{3}$} \\
\hline Camp clinic & 106 & 77.4 \\
\hline Community Health Worker & 37 & 27.0 \\
\hline Private clinic & 51 & 37.2 \\
\hline Pharmacy & 36 & 26.3 \\
\hline Shop & 4 & 2.9 \\
\hline Others & 29 & 21.2 \\
\hline \multicolumn{3}{|l|}{ Perceived correct dosage for ECs } \\
\hline One tablet a day & 25 & 18.2 \\
\hline Before Sex & 12 & 8.8 \\
\hline Immediately after unprotected sex & 18 & 13.1 \\
\hline One tablet initially, one 12 hours later & 4 & 2.9 \\
\hline Used every morning & 10 & 7.3 \\
\hline Not sure & 15 & 10.9 \\
\hline Others & 53 & 38.7 \\
\hline
\end{tabular}

$1=$ Missing information; $\mathrm{n}=926$

$2=$ Missing information; $\mathrm{n}=132$

$3=$ Multiple responses; $\mathrm{n}=137$; Column total does not add to 100

$4=$ Family planning staff, workshops, TBAs among others

Nearly all women (100/102) who did not respond to this question were Sudanese. Among the remaining Sudanese women $(\mathrm{n}=367), 63.5 \%$ were unaware about any preventive measures to avoid pregnancy though $24.8 \%$ and $6.5 \%$ reported going to a hospital or emergency contraceptives as possible options respectively. Among the Somali refugees who responded to 
this question $(n=294)$, though $50.0 \%$ were unaware of any options, the knowledge regarding opting for emergency contraceptives $(10.2 \%)$ was higher than among the Sudanese.

Education was a key factor in knowledge regarding emergency contraceptives. Nearly $65 \%$ of women, who were aware of emergency contraceptives as a measure to avoid a potential pregnancy following unprotected intercourse, had received secondary level education. Majority of the women (41.6\%) who were unaware of any preventive measures to avoid pregnancy were illiterate. Most of the women (48.3\%) who were aware of emergency contraceptives were younger women $(20-29$ years $)$.

Nearly $15 \%$ of women (137/926) claimed to have ever heard of EC. Most were Somali $(48.2 \%)$ and Sudanese $(37.9 \%)$, in the younger age group of $20-29$ years $(43.8 \%)$ and had received secondary level education (52.3\%). Nearly half of them (48.5\%) had heard about EC in the recent past (past five years).

Women mostly reported that they had heard about EC from the clinic (59.9\%). Some of the other sources of information identified included friends/family (30.7\%), newspaper/magazines $(11.7 \%)$ and family planning staff (7.3\%). Most of the 137 women $(77.4 \%)$ were aware that ECs could be obtained from the camp clinics. Other reported sites where EC could be obtained included private clinics $(37.2 \%)$, community health workers $(27.0 \%)$ and pharmacy $(26.3 \%)$. While Somali women more often reported ever hearing about EC as compared to Sudanese women (48.2\% and 37.9\% respectively); Sudanese women more often were aware where to obtain ECs - camp clinics $(35.8 \%)$, community health worker $(16.8 \%)$, private clinics $(25.5 \%)$ or pharmacy $(16.8 \%)$. [Table 3]

Though being aware of the method and where to obtain ECs, none of the 137 women correctly reported its usage - neither in the number of tablets or when to start taking ECs. One tablet a day $(18.2 \%)$, used every morning $(7.3 \%)$, immediately after unprotected sex $(13.1 \%)$ or before sex $(8.8 \%)$ were some of the frequently reported responses. Furthermore, equal numbers of women reported that ECs would work $(n=54)$ or not work $(n=54)$ when a woman recognizes delayed menstruation. However, Sudanese more often reported that ECs would work when menstruation is delayed (20/54) while Somali women reported more often that ECs would not work when menstruation is delayed $(27.0 \%)$.

Table 4: $\quad$ Percentage distribution of knowledge regarding emergency contraceptives mode of action $(\mathrm{n}=137)$ 


\begin{tabular}{|l|rc|}
\hline \multicolumn{1}{|c|}{ Characteristics } & $\mathrm{n}$ & $\%$ \\
\hline Will EC work for delayed menstruation & 54 & 39.4 \\
Yes & 54 & 39.4 \\
No & 29 & 21.2 \\
Not sure & & \\
\hline Perceived rate of success for ECs & 54 & 39.4 \\
Almost 100\% & 38 & 27.7 \\
$75 \%$ & 24 & 17.5 \\
$50 \%$ & 8 & 5.8 \\
$25 \%$ & 13 & 9.5 \\
Not sure & & \\
After unprotected sex, when should EC be used & 53 & 38.7 \\
Immediately afterwards & 29 & 21.2 \\
Within 24 hours & 36 & 26.3 \\
Within 72 hours & 7 & 5.1 \\
Within one week & 2 & 1.5 \\
Anytime before the next menstrual period & 10 & 7.3 \\
Not sure & & \\
Perceived composition of ECs & 48 & 35.0 \\
Same as regular contraceptive pill & 48 & 35.0 \\
Stronger than regular contraceptive pill & 25 & 18.2 \\
Completely different & 16 & 11.7 \\
Not sure & & \\
\hline
\end{tabular}

Though nearly $40 \%$ of the 137 women who were aware of ECs perceived a success rate of $100 \%$, another $27.7 \%$ reported a $75 \%$ success rate. However, exact knowledge regarding timing of EC ingestion following unprotected sex was not high. Only $26.3 \%$ of the 137 women reported within 72 hours, though $38.7 \%$ and $21.2 \%$ reported immediately afterwards or within 24 hours respectively.

Perceptions regarding the content of ECs were exceptionally good (either same $(35.0 \%)$ or stronger (35.0\%) than regular contraceptive pills) though $18.2 \%$ reported that it was completely different from regular contraceptive pills.

Attitude towards Emergency Contraception [Table 5]

Though by far most of the women interviewed reported that they considered ECs good $(71.9 \%)$; a few women reported that it was bad (7.4\%). Other comments made were more specific such as helps prevent unwanted pregnancy $(6.8 \%)$, good for rape/unprotected sex (1.4\%) among others. However, $22.9 \%$ of the 926 women interviewed reported concerns with EC use. These included "cause health problems to the mother" (12.7\%); "harm the baby" $(2.4 \%)$, "not legal" (22.2\%) among others. Interestingly $49.6 \%$ of the 137 women who reported that they were aware of ECs had reservations regarding ECs. Despite voicing their concerns, most of the women interviewed either strongly agreed $(44.2 \%)$ or agreed $(38.3 \%)$ that EC is suitable for women in a refugee status. 
Table 5: $\quad$ Percentage distributions of attitudes regarding emergency contraceptives

\begin{tabular}{|l|rr|}
\hline \multicolumn{1}{|c|}{ Characteristics } & N & $\%$ \\
\hline Perceptions regarding EC & & \\
Good & 764 & 71.9 \\
Bad & 79 & 7.4 \\
Helps prevent unwanted pregnancy & 72 & 6.8 \\
Helpful / useful & 46 & 4.3 \\
Good for rape / unprotected sex & 15 & 1.4 \\
Made available & 3 & 0.3 \\
Helpful to refugee women & 9 & 0.8 \\
Not sure / don't know & 14 & 1.3 \\
Other & 17 & 1.6 \\
\hline Variety of concerns regarding EC use ${ }^{2}$ & & \\
Not yet familiar & 92 & 43.4 \\
Health problems & 27 & 12.7 \\
Harm the baby & 5 & 2.4 \\
Affect future fertility & 14 & 6.6 \\
Is an abortion & 5 & 2.4 \\
Illegal & 47 & 22.2 \\
Predispose to STIs or HIV & 11 & 5.2 \\
Men will pressure women to use ECs & 15 & 7.1 \\
Abused by women as a contraceptive method & 6 & 2.8 \\
Others & 30 & 14.2 \\
\hline Spousal Opposition & & \\
Yes & 349 & 38.1 \\
No & 318 & 34.7 \\
Not sure & 249 & 27.2 \\
\hline
\end{tabular}

$1=$ Multiple responses; $\mathrm{n}=926$; Column total does not add to 100

$2=$ Multiple responses; $\mathrm{n}=212$; Column total does not add to 100

$3=$ Missing information; $\mathrm{n}=916$

The majority of women suggested that ECs should be offered either by doctors $(93.6 \%)$, staff of a health facility $(73.7 \%)$ or community health worker/CBD $(67.6 \%)$. Though $27.9 \%$ of women felt that ECs should be available to anyone on request, others clarified that ECs should be available only to married women $(16.4 \%)$ or women who have been raped (17.3\%) but not to young women $(27.2 \%)$. However, most $(71.0 \%)$ reported that they would either use it themselves or recommend use of ECs to friends.

Spousal opposition to EC use was reported by $34.7 \%$ of women. Of the 349 women who reported no spousal opposition, the majority $(47.6 \%)$ was Sudanese. Female education did not appear to play a role in the perceptions of spousal opposition as our results indicate that 
approximately equal proportions of refugee women reported spousal or no spousal opposition irrespective of their education categories ${ }^{\mathrm{g}}$.

The outlets for EC information dissemination recommended were mainly via community health workers $(44.1 \%)$ or service providers $(30.2 \%)$ though respondents also mentioned women support groups (17.9\%) and meetings with women (13.4\%) (see Table 6 below). Sudanese women more often suggested health care providers than Somali women ${ }^{\mathrm{h}}$ though approximately equal proportion of Sudanese and Somali women reported women support groups or meeting with women ${ }^{\mathrm{i}}$.

Table 6: $\quad$ Percentage distributions of knowledge regarding emergency contraceptives

${ }^{\mathrm{g}}$ No spousal opposition: Illiterate 34.9\%; Grades 1 - $530.3 \%$; Grades $6-1234.1 \%$ Spousal opposition: Illiterate 35.0\%; Grades 1 - $524.9 \%$; Grades $6-1236.9 \%$

${ }^{\mathrm{h}}$ Community health workers - Sudanese (52.7\%), Somali (37.3\%); Service providers - Sudanese (41.5\%), Somali $(11.5 \%)$

${ }^{\mathrm{i}}$ Women support groups - Sudanese (20.6\%), Somali (19.3\%); Meetings with women - Sudanese (11.8\%), Somali $(10.5 \%)$ 


\begin{tabular}{|c|c|c|}
\hline Characteristics & $\mathrm{n}$ & $\%$ \\
\hline \multicolumn{3}{|l|}{ Information Dissemination $^{1}$} \\
\hline Community Health Worker / Education & 404 & 44.1 \\
\hline Service providers & 277 & 30.2 \\
\hline Traditional Birth Attendants & 176 & 19.2 \\
\hline Health Facilities & 123 & 13.4 \\
\hline Women support groups & 164 & 17.9 \\
\hline Meeting with women & 123 & 13.4 \\
\hline Community leaders & 56 & 6.1 \\
\hline IEC & 22 & 2.4 \\
\hline Others & 87 & 9.5 \\
\hline \multicolumn{3}{|l|}{ Facility where EC should be available $^{2}$} \\
\hline Doctor & 864 & 93.6 \\
\hline Any staff of a health facility & 680 & 73.7 \\
\hline Pharmacy & 530 & 57.4 \\
\hline Community Health Worker or CBD & 624 & 67.6 \\
\hline Local shops & 189 & 20.5 \\
\hline \multicolumn{3}{|l|}{ Clientele of $\mathrm{ECs}^{3}$} \\
\hline Available to anyone & 258 & 27.9 \\
\hline Not young girls & 252 & 27.2 \\
\hline Only married women & 152 & 16.4 \\
\hline Only women who have been raped & 160 & 17.3 \\
\hline Only women who report contraceptive failures & 13 & 1.4 \\
\hline Others & 209 & 22.6 \\
\hline \multicolumn{3}{|l|}{ Recommend for use $^{4}$} \\
\hline Yes & 655 & 71.0 \\
\hline No & 155 & 16.8 \\
\hline Not sure & 112 & 12.2 \\
\hline
\end{tabular}

$1=$ Multiple responses; $\mathrm{n}=916$; Column total does not add to 100

$2=$ Multiple responses; $\mathrm{n}=923$; Column total does not add to 100

$3=$ Multiple responses; $n=926$; Column total does not add to 100

$4=$ Missing information; $\mathrm{n}=922$ 


\section{B. QUALITATIVE COMPONENT}

\section{Knowledge on Contraception including Emergency Contraceptives:}

The moderator sought to know whether the participants were knowledgeable of the ways to prevent pregnancy. Most of the participants knew about pills, condoms and IUD. They indicated that they had heard of these from a variety of sources including FP clinics, teachers in school and from general discussions within the village.

The mixed adolescent male discussion groups were particularly familiar with the various contraceptive methods, having attended reproductive health seminars that are organized within the camp by National Council of Churches of Kenya (NCCK) as part of their reproductive health programme. However the girls were not too familiar with these methods. This is in line with the findings of an earlier survey undertaken by IRC in the camp which revealed that $35 \%$ of women in the camp were able to cite any modern methods of contraceptives, and only $36 \%$ knew that contraceptives were available at the camp health facilities. Current use of modern contraceptives among women in the camp then was only $8 \%$. The current survey has shown use of modern contraceptives to be $23.4 \%$.

Asked where they would get more information on FP, the participants generally cited clinics. A majority of the participants in the various groups had not heard about EC even though it is available at the camp hospital. However among the Somali male youth, one of them had a rough idea of EC as he mentioned that there are tablets specifically available for prevention of pregnancy. He said that he got the information from magazines and television. It is at this juncture that the moderator indicated that she would share more information on emergency contraception.

\section{Introduction of Emergency Contraceptive}

The moderator explained that emergency contraception is a term used to describe methods of contraception that women can use after unprotected sexual intercourse to help prevent pregnancy. For example, birth control pills used in a higher dose and taken within 72 hours after unprotected sex works well as an emergency contraception. Sometimes this therapy is called "morning after pill".

Emergency contraception pills must be started within 72 hours after unprotected sex and they are about $75 \%$ effective. The ingredient is the same as the regular birth control pill, but a larger dose. Some women who take it experience relatively minor side effects such as nausea and vomiting. Emergency contraception is used widely overseas, for example in England and in the United States of America. Although it is possible to get emergency contraception in Kenya and other African countries it is not yet widely known. From the name, the method is for contraceptive emergencies and not for use as a regular method.

Having introduced EC, the participants initially commonly confused it for an abortifacient. For example one said that the drugs might be harmful to the baby to facilitate abortion. At this point the moderator set out to share more information on EC by reiterating that it is not for FP but emergency for prevention of pregnancy. Furthermore it is neither an abortion, as conception has not yet taken place given the time frame within which it should be taken. 
She also explained that like any other method EC has limitations in that those women who have a heart condition cannot use it. In terms of efficacy it was further explained that out of every four women it works for only three i.e. an effective level of 75 per cent.

One of the participants wanted to know how long it takes for one to get pregnant, having used the method? It was explained that the method works normally by preventing a pregnancy that may occur following unprotected sexual intercourse, after which a woman resumes her normal menstrual periods and can therefore is at risk of a subsequent pregnancy. At this point one of the male respondents acknowledged very keenly "That is a very good medicine, what is the name"? Acknowledging its apparent simplicity in use, yet another male participant suggested that "it should be advertised everywhere!"

The moderator reported that there are different brands of emergency contraception including postinor and PC4. However, in Kenya Postinor is the most commonly used. As to which is the best they were informed that both are equally effective but like any other medicines there are side effects.

More questions from participants revolved around the issue of having unprotected sex and whether it is in order to use Emergency Contraception as often. They were reminded that if sex is frequent then it is important to use a regular method of family planning as Emergency Contraception is meant only for emergency and not for every day use.

\section{When should women use Emergency Contraception?}

For most contraceptives the timing is crucial to ensure effectiveness of the method. This is even more so for Emergency Contraception that is to be taken within a specified time frame for it to be effective. From all the participants in all the discussion groups, it was not readily clear when women should use Emergency Contraception. A variety of responses were elicited notably after sex, before sex or when one realizes that one is pregnant. Some of the participants suggested that it should be before fertilization, others suggested immediately after sex and yet another said within 20 and 21 days! These responses provided some further insights and confirmation that there is little knowledge about EC and how it works. However as to whether it works always: one participant responded in the negative while another observed that it might not work especially if the time "expires". In yet another instance of misconception, a participant said it would not work if one were already pregnant. The men were equally ignorant as to how it works and at best seemed to regard EC as an abortifacient. This is evident in the following excerpt of the discussions among the Somali men:

Participant: But fertilization has occurred?

Moderator: No. It has not occurred. If fertilization occurs it will not work. It is supposed to prevent fertilization but can only work within 72 hours. This is why when we get rape victims coming to hospitals it is one of the points that we make sure.

Participant: How long does fertilization take place?

Moderator: Five days

Participant: I thought that it takes place immediately.

Moderator: No, it takes five days.

Participant: We have believed that it is immediate since the egg if there?

Moderator: It is not immediate. It takes upto 72 hours for the sperm and the egg to meet nicely and get together to form a special type of a coating around the fertilized egg. The fertilized egg has to move to be implanted somewhere for proper germination. This takes more than one week. So if you want to prevent fertilization you should take it within 72 hours and this is why it is called emergency contraception. Sometimes people used to think that it is abortion. But 
abortion means that the egg has already been fertilized and you have to remove it. It means that if you are using EC after 72 hours.

Determined to convince himself that emergency contraception within 72 hours of unprotected sexual intercourse is not abortion, one participant quipped "You are not killing as that is a sin you are removing" (general laughter). The moderator further reassured him that it is not killing because it is not effective in terminating an established pregnancy but is effective in preventing fertilization. She reiterated that it does not remove the fertilized egg but rather prevents the egg from fertilization. It ensures that the environment is not conducive for fertilization to take place. The moderator further elaborated the method - for EC to work one has to take it within three days. There are only two tablets to be taken at an interval of 12 hours.

The moderator, however, cautioned that the Emergency Contraception does not always work for every woman. Out of every four women it will only work for three women. Because of the apparent misconception and therefore discomfort over Emergency Contraception being misconstrued as an abortifacient it was crucial to establish under what circumstances the participants felt that Emergency Contraception should be used.

A majority indicated that this is an appropriate method for one that gets pregnant after rape or if one has been tricked into sex. This was based on the acknowledgement that rape was very common in the camp or that one could easily be tricked into sex and get pregnant. Even the elders who were at first uncomfortable with the issue conceded that this is a useful method in respect of rape.

The Somali elders were at the end very convinced after the explanation on how Emergency Contraception works. This was so to the extent that one asked "Can I use it for my wife if I don't want her to get pregnant? Is it for married couples"? He had to be reminded that this is only for emergency. This was also a recurrent question among the youth that noted that it as " $a$ very good method" and were equally keen to know how often it can be used. They were again reminded that this is only for emergency and not for frequent use.

\section{Concerns on Emergency Contraception:}

Contraception in general has generated controversies, often suspicion and fears within the recipient community. As a new method in the community, it was important to establish from the onset whether the participants would have any concerns or fears about the EC method. The adolescent women had an idea of some of the side effects. These included such side effects as "pain in your stomach, allergic to the pills while another suggested vomiting and nausea.

In contrast and understandably so, the male youth and elders could only raise questions as to whether there are any side effects. At this point the participants had more questions and issues of clarification with regard to the method rather than respond directly to the moderator's question.

One participant wanted to know whether EC could be used always in the same manner as the other contraceptives? In response the moderator gave an analogy of an accident indicating that $\mathrm{EC}$ is for emergency only and this should not occur every day.

The moderator also emphasized that this is a very high dosage given that one of the tablets is the equivalent of 8 tablets of the normal contraceptive pills. On the same subject another participant wanted to know whether EC could be used again for example in a situation where 
one is raped. She reiterated that this should only be used on an emergency basis. Situations that would lead to such an emergency should be avoided - it would be too unfortunate to have another accident in the same week!

The participants also wanted to know the advantages and disadvantages. The moderator stated that the main advantage is to avoid pregnancy. The disadvantages are: that it may not always work, it has a time limit in that it has to be taken within three days of the sexual intercourse, it is a high dose and may therefore cause discomfort and so it is important to take it after a meal. Furthermore one cannot use it everyday, as is the case with the regular contraceptives.

In terms of whether the participants thought EC would be useful in Kakuma this was acknowledged to be the case as one male participant reported "I have observed many cases of rape in the camp". However, in the same breath he cautioned that the old people in the camp cannot agree for it to be introduced in the camp and this may also be the case with the uneducated or ignorant members of the community. A cause for alarm was that women might misuse it to avoid giving children to their husbands. This was particularly the case for Sudanese men for whom childbearing is of great importance as they feel that those who have died in the war must be replaced. Another fear that was expressed is that Emergency Contraception " will increase the rate of playing sex in the camp" and so it was important to take necessary precautions when introducing Emergency Contraception in the community.

\section{$\underline{\text { Views and Attitude towards Emergency Contraception }}$}

Generally many communities have not readily accepted modern methods of contraception as these have been viewed as a deliberate means of controlling fertility. At best they make reference to culturally acceptable ways of child spacing. For example among the Sudanese, traditionally, the childbearing varied from family to family, resource power and incidence of child mortality. They argued that children are weaned depending on how much resource the family has at its disposal. The more the resources, the more children one may get. A woman's diet is very important and is related to her reproductive ability. If the woman is healthy due to the food she takes, she can reproduce many children. Over and above this, sometimes there is a possibility that only two or three children out of ten in the family survive. For this reason many children are born to ensure that if some do not survive, the family will be left with some to ensure continuity of the lineage. Currently the group also recognized that generally availability of modern health services determines the number of children to survive. The concern with child survival is partly the reason for the practice of polygamy and the reluctance to accept modern contraception.

Having discussed at length about EC, the moderator sought to know what the different groups thought of the method and whether it would be useful to introduce it in Kakuma. Across the groups it was conceded that this is a useful method. One of the Somali men was very categorical and stated the following with regard to EC: "Really, it is a good idea. It is very good". Another male participant justified its usefulness by stating that "I have many cases of rape in the camp".

All the Somali girls responded in the affirmative and one participant who is 14 years old said, "most girls are doing sex" while another said " 14 year olds are getting pregnant" and this seemed to be a major concern for them. It was considered particularly important for Kakuma noting that rape is a frequent occurrence and it rendered women helpless in that they had no way out. 
In all the discussion groups, gender violence including rape were acknowledged as a common feature within camps. However, at first there was denial of rape among the Somali elders but as the discussion progressed, it was clear that this is a problem they grapple with frequently within the camp regardless of who is the perpetrator. One of the participants stated that rape is very common in Kakuma.

The Somali elders confirmed this but attributed the vice to other groups and not the Muslim community. In defense of their culture one of the participants said:

"We have not seen such things. If a girl does not accept... this is rape, but we have not seen these things in the camp. We don't allow our girls and boys to go to school places together so that chances of them meeting is minimized. This is very rare in our culture and religion".

However, another participant said:

"I know a lady who was raped in our community last year. Some people were waiting for her at night. Three men came to her house. She came out for a private call. They caught her mouth. She was raped, taken to the hospital. I don't know whether or not she was given this tablet".

Asked what happens when one is raped, one of the Sudanese girls said, "You get married if the man is known". The Sudanese boys and the mixed adolescent group shared this sentiment. One male participant noted that although he had not come across a raped woman he was aware that women whose pregnancy results from rape are physically and mentally affected and will never forget the fact that she cannot know who fathered the baby. One of the female respondents qualified this observation as follows "She can kill the child" while a male stated that "She can kill herself." Being aware of the war situation in Eastern Europe another male participant stated:

"The soldiers have raped most of the Bosnian women that I have heard of. So all the women started killing the babies first because they had been raped and do not want to protect these babies".

Given the traumatic experience associated with rape, the group regarded EC as the best solution under the circumstances. Similarly among the Somali youth it was revealed that when a girl is raped, gets pregnant and she is not married it is shameful and therefore she might even not tell her mother, aunt or neighbour about it even when it is clear that it is not her fault. It was noted that:

“... They get mentally disturbed, depressed and can even commit suicide. Some can wait for the baby to be born so that she kills the baby. It is very hurting and she keeps remembering".

Thus, in the context of emergency, the participants had no problem in embracing EC that seems to have come at an opportune time.

\section{For whom should Emergency Contraception be available?}

In many communities contraceptive use has been acknowledged, though grudgingly and for a limited category of women mainly those who are married. Contraceptive use among unmarried women is generally deemed inappropriate. The Muslim community viewed contraceptives in 
the context of promiscuity and generally against their culture while the Sudanese did not see the relevance of contraceptives in their situation where so many lives have been lost and therefore need to be replaced.

EC as a method for use during emergency was acceptable to the various groups particularly to the elders who were often conservative and even uncomfortable with the idea of contraceptives in general. Having acknowledged the incidence of rape within the camp and its consequent problems, EC was a welcome solution. While acknowledging the potential usefulness of EC, some groups had concerns and felt that EC should be earmarked for a specific group of women. It was clear that it should not be given to everybody except for those with accidents especially rape.

The youth were more flexible and although the girls stated that it should not be given to girls who have not yet started their menses they also stated that it is a method they would recommend to a friend in need. In the boys' discussion groups, they were particularly enthusiastic about contraceptives in general. They wanted to know details about safe periods and it was clear that they were keen to protect their partners. They were highly supportive of EC and for them the method should be widely available to meet the reproductive needs of women.

\section{Where should Emergency Contraception be available?}

In Kenya all types of contraceptives are generally available at health facilities. However oral contraceptives are available through community-based distributors. A combination of these channels makes accessibility of contraceptives easier and therefore ensures large population coverage. Currently EC is only available at the camp hospital. The participants indicated that EC should be available in a variety of places including the clinics and pharmacies in Kakuma town. However, they were very emphatic about the fact that these cannot be stocked in shops, as "the one in the shop cannot tell you how to use the method". Somali elders were particularly concerned about where EC should be available to counter abuse. This was based on experience regarding other medications that were reported to be readily available within the community and often sold without any controls.

On the issue as to where to store EC, some suggested at home for ease of use while others thought it is too risky as they can be taken by anybody by mistake. It was finally settled that they be kept at clinics as the most appropriate place and be administered by a specific person to curb any possible misuse. This was based on the notion that this is like other medicines that are routinely kept in the hospital and therefore not everybody in the community handles them. However, it was also observed that personnel who work in the hospital could get the medicine and give or sell to people. Among the male participants, the Somali men observed that:

"Medicine in the hospital is sold in the camp by hawkers they ask, "do you want medicine, do you want capsules? They have sacks with a lot of medicine, so everything in the camp can be gotten very easily... They come from the hospital and I know it is a big problem, just if you want syrups you can buy it at ten shillings from those people who roam in the camp. Instead of going to the clinic looking for this drug just buy it. We know it is from the hospital because of its price. Some come from Sudan and the price is very low".

For this reason, the men emphasized that EC has to be monitored closely, by tracing how many have been given out. This was reiterated in the mixed boys' discussion group where it was stressed that to facilitate access, EC should be available at the clinics because every zone has its 
own clinics while the hospital is only for referrals. Within the clinic there should be a special place for dispensing and trained staff should man this. More importantly the clinic personnel should carefully assess the potential clients for EC prior to dispensing it to avoid misuse and family problems. There should be monitoring and evidence that it was indeed an emergency.

For those clients who may be shy to access this at the health facility it was suggested that community health workers could be the source of EC. This would protect many women who are afraid of being stigmatized as using the pills in a community that is still conservative in the midst of dramatic social change. It was noted that some women could not say that they were raped or the condom burst. One Somali male participant observed that: "In Somali traditions one cannot say this, they should rather die or go to Kakuma Mission". This category of people therefore requires more personalized attention in terms of working out the most feasible mode of service delivery.

\section{Who should be informed of Emergency Contraception and How?}

Specific groups use contraception, by its very nature. However, in terms of advocacy and information dissemination it is imperative that contraceptive information be made available beyond the specific groups. This will facilitate acceptability and the decision-making process regarding use of contraceptives. Generally, the participants felt that all members of the community should be informed about EC, more so "the young generation" and adolescents.

Mothers were also mentioned as a special group that needs information regarding EC. Somali men observed that: as they are the ones who bear the consequences and the stigma that comes with rape or any form of sexual violence, it is therefore vital that they are well informed about EC.

It was stated that it is particularly important that members of the local security team within the camp be informed about EC and where it can be obtained. This is because they are often the first to come in contact with rape victims and are usually at a loss as what to do next apart from simply reporting the case to the administration. Leaders of women support groups should be informed about EC, information which they should share among themselves and sensitize their group members "so that if any woman comes across such an accident she may run to the hospital even before telling others."

All the participants agreed that the women should know about EC and were also enthusiastic about telling others about it. The moderator cautioned them that although their idea of spreading word is good, it is important to wait until they are adequately prepared with full information about the method. But one male participant insisted that "for the time being I will tell them that there is medicine for rape."

The Somali girls suggested that adolescents be informed through youth meetings and peers groups. Posters were also suggested but one of the participants thought that this would be too embarrassing, The rest of the participants conceded that this might not be the best way to communicate. This was supported by the views generated from the mixed Sudanese and Rwandese boys who were of the opinion that basically such information should be obtained from the health facilities.

Given that the youth are already involved in dissemination of information within the camp, they should therefore be one of the channels for disseminating information about EC. In addition, it was suggested that the information should be made available to the schools and adult education classes so as to reach both youth and adults. 
To ensure that the information reaches the various target groups, the boys suggested organization of seminars for different groups. The youth groups who were already involved in puppetry and drama entertainment sessions suggested that they could easily incorporate information on EC in their activities. It was further suggested that posters and billboards could be placed in schools, public places, and community centres.

Participants suggested various avenues that can be used to create awareness and use of EC including introduction in the hospitals; training of youth as dispensers; mobilization of the community through drama, puppetry by the youth and holding workshops for women and girls as direct users. The participants asked for the moderator's opinion but she emphasized that the approach must be people driven from within.

It was therefore acknowledged that due to the diversity of interest groups and stakeholders it would be good to have a combination of approaches including separate strategies for youth, adults, literate, and illiterate. Some of the existing avenues within the camp include a reproductive health magazine that can incorporate these issues. Group meetings are popular among both gender and were therefore suggested as appropriate avenues for the dissemination of information on EC.

At the conclusion of each of the eight focus group discussion sessions, time was devoted to answering any of the concerns of the participants. Some of the concerns raised were:

Female participant: I am pregnant-I have a child and I would like to rest for 1 to 2 years after I deliver. Can you advice me?

Moderator: Go to the clinic and you will be given a method that is good for you.

Participant: $\quad$ What is the legal basis of this EC

Moderator: This is not an abortion pill, it is a medicine (contraceptive) manufactured and registered in Kenya, so it is acceptable.

Male participant: $\quad$ Does it interfere with the uterus?

Moderator: No

Male youth: $\quad$ What will happen if one uses it often/frequently?

Somali male youth: Is there a pill for men?

Moderator: $\quad$ There are no pills for men. Everything is for women, one day we will have to invent one for men.

The boys were given an explanation about calendar checking and counting as a method of regulating fertility, about unsafe and safe days. They were enthusiastic to know more about this method.

Questions raised specifically by the youth included:

Somali male youth: How does one know that one has gonorrhea?

We were told that aspirin and panadols work. Is it true?

Sudanese girls: $\quad$ Why do some women not resume their menstrual cycle after having a baby for some time?

Why do some stay before having sex for as long as two years?

What does it mean when a girl has her periods before the days are due?

Some people say that if one has sex with the man and she is pregnant the baby can come out. Is this true? 


\section{DISCUSSION}

\section{A. QUANTITATIVE COMPONENT}

\section{i) Health Care Providers}

The categories of Reproductive Health service providers accessible to the refugee women living in the refugee camps designated as Kakuma I, II and III included physicians working in the camp hospital located in the largest of the three camp sites (Kakuma I), clinical officers, nurses and family planning service providers. The main reproductive health service provided by clinical officers, nurses and family planning service providers were family planning counseling and supplies. These were restricted to hormonal methods and condoms. At the camp hospital, additional RH services including those for STIs, pregnancy testing, ECs and MVAs were offered. None of the HCPs interviewed reported stock-outs for contraceptive supplies. Based on these findings and the ease of availability of the health services to the refugee women, the low acceptance for contraception among the refugee population has lead to about $23.4 \%$ of the refugee women ${ }^{j}$ reporting being current users of a contraceptive method.

With regard to the type of RH services offered, it was interesting to note that information and dispensation of ECs were generally under the management of the doctor or nurse in the camp hospital despite the high level of awareness of ECs $(81.3 \%)$. Though the reasons for prescribing ECs were generally appropriate (prevention of pregnancy $(46.2 \%)$, good for rape victims $(30.8 \%)$, some of the HCPS reported certain misgivings regarding ECs including against religion/culture (7.7\%) or an abortifacient (15.4\%). Additional concerns regarding ECs use were the several questions raised by the HCPs regarding unprotected sex, feasibility of introducing this method into the community among others. It is important to point out here that, based on our findings, misconceptions regarding use of ECs, mode of action and correct administration of ECs will need to be clarified in the training program.

The results of this baseline survey among the HCPs indicates that though the provision of ECs was currently restricted to the doctor and nurses based at the camp hospital in Kakuma I, but there was no consistency in type, dosage and timing for the provision of ECs, no service delivery protocol or IEC materials that the clients could browse through or be counseled with. Hence, as part of the overall intervention project, we suggest that training be focused on appropriate regimens for ECs, counseling and provision of IEC materials. The notion of lack of IEC material depicting reasons for provision of ECs was mentioned again when the HCPs were questioned regarding low acceptability for ECs.

Another aspect based on our findings that needs to be considered with the input from the camp health officials is expanding the prevailing policy on dispensation of ECs by the doctor or nurses to include all HCPS who supply contraceptives. In addition, based on our findings regarding the advance distribution of ECs among the refugee women, we suggest that this needs to be discussed with camp officials and refugee elders. Suggested channels for dissemination of information regarding ECs included health facilities (56.3\%), home visits $(50.0 \%)$ or through TBAs $(12.5 \%)$. Although we suggest that, based on the reported cultural

\footnotetext{
${ }^{\mathrm{j}}$ See Report on Refugee women in the Kakuma camps
} 
$(18.8 \%)$ and religious (12.5\%) barriers to contraceptive use, discussions with camp officials and camp elders will greatly facilitate the acceptance of this method for unprotected sex, condom rupture, or rape among others.

\section{ii) Women}

Sudanese (50.5\%) and Somali (32.0\%) refugee women from Kakuma I camp were the major respondents from the refugee camps designated as Kakuma I II and III. They were young ( $40.7 \%$ in their twenties) and nearly $67 \%$ of them had received at least primary level education. Current use of contraception was $23.4 \%$ with pills and injectables being the most commonly reported modern methods used.

Lack of knowledge regarding the rationale for emergency contraception was a significant finding of the survey. A large number of women (56\%) reported that there was no preventive measure that could be adopted following unprotected sexual intercourse. Only $7.3 \%$ mentioned emergency contraceptive as a preventive measure. In addition, among the women who knew about emergency contraception, none of them correctly reported its usage in terms of number of tablets or when to take them though most of them (70\%) were correct in their understanding of the content.

In conclusion, the key findings from this study reflect the lack of knowledge regarding emergency contraception. In the refugee community where the prevalence of rape was reported to have increased in the recent past, it is significant that most of the women interviewed were unaware of EC as a preventive measure. However, the majority of the respondents not only reported that ECs were suitable for women in refugee camps but also were interested in learning about ECs and recommended health care professionals [staff at health facilities or community health workers] or women support groups as the outlets for information dissemination. 


\section{B. QUALITATIVE COMPONENT}

In all the discussion groups a recurrent theme was the dilemma between culture and modernity. From these groups it was clear that as refugees, their societies experience change that often is in conflict with their culture let alone the traumatic experience of displacement. The sociocultural dictates and expectations are distinctly clear between the older people and the youth and yet this was hard to practice. For example the mixed group of Sudanese adolescents reported that rape is rare in traditional Sudanese culture but they were also quick to point out that when it happens "a raped girl cannot report and traditionally this rarely happens among the Sudanese.... If pregnant the girl just goes to the boy's home”.

Two female participants indicated that most girls among the Dinka do not indulge in premarital sex as a very high value is placed on virginity. Thus, breaking the rules is shameful and the height of irresponsibility and "she may bring in diseases". However, they further acknowledged, "now we are in Kenya things might have changed".

A majority of the respondents felt that within the multi-social and cultural setting they find themselves operating in situations that are not only counter to their cultural expectations but also have negative implications. This is also in line with the observation of The Interagency Field manual for Reproductive Health in Refugee Situations which states that "sexual and gender-based violence is strongly associated with situations of forced population movement. In this context it is vital that emergency post-coital contraception supplies are available to those who request this."

In all discussion groups rape featured as an important reproductive health concern. The girls acknowledged that EC would provide them an alternative. They would not have to yield to a situation of helplessness where the only recourse to rape and the resulting pregnancy is either an abortion or marriage to the man if he is known, notwithstanding their stand on the matter.

For the Muslims and the Sudanese, the notion that women and girls should avoid moving at night or their brothers should accompany them even if one is married was only nostalgic. These perceived practices could no longer hold since the social structures in which they are meant to function have already broken down, as most refugees do not comprise complete families.

There are many unaccompanied youth; estimated at between 3,000 and 4,000 in the Kakuma refugee camps. Some of these are girls who may be taken care of by other families or relatives but at best one would not expect the young women to get the protection that would normally be accorded in a traditional social setting. Many unaccompanied boys end up setting-up their own households within the camp under the care of an elder but on the whole they operate as independent individuals.

In terms of attitude towards contraceptive use in particular and EC in general one can discern a generational conflict. Whereas the youth were more accommodating, the adults were at first adamant and often on the defensive. On the whole the youth had a more favourable attitude towards FP than the adult men did. Noting the position of the adults and given the fact that they influence decision-making, FP is not very popular within the camp or across ethnic groups.

Among the Muslims, the practice of FP was seen as interfering with procreation and encouraging promiscuity. On the other hand, the Sudanese felt that natural family planning is adequate. More importantly, under the circumstances, the Sudanese felt that FP among them would be a misguided effort. The Sudanese girls' position is illustrated by: "We do not know the reasons but our fathers do not want FP. They say our people are dying then why do you practice FP? They want to replace the dead". 
In the Sudanese male discussion group, it was emphasized that the concept of an unwanted pregnancy and consequently child does not exist. It was noted that even a child resulting from adultery is accommodated. However, the male adulterer has to pay a fine for his actions. As one participant explained:

"The concept here is not enjoyment of sex, but for procreation. So every time a man and a woman have sex, they have already planned for a child. That is why some men have many wives to get many children and this is prestige in itself. In a situation like where there is war in Southern Sudan, families are having many children because their own people have died in the war".

This is how many of them perceived it to be and were therefore ready to dismiss it outright.

It was, however, acknowledged that due to resource constraints child spacing using modern methods was taking place in the camp at various levels of intensity. Besides, these methods are readily available but as a community, they have no control over their use. The Sudanese men also noted that they have had a lot of training in reproductive health. They also acknowledge that they now live in a different environment with different views and cultures from their own. With regard to FP they felt with the changing times, at least people have a choice and can choose to use it either secretly or openly so long as there is a protection base that is in the long run positive. For this reason it is important that the way EC is introduced in this community should underscore upfront its usefulness as an emergency measure. A very clear explanation that indeed it is not for family planning is likely to yield positive results.

Whereas the traditional structures have disintegrated, these have been replaced by adaptive social structures that are not only protective but also serve as social control mechanisms and vehicles for settling disputes. The role of elders within the dominant refugee groups i.e. the Sudanese and Muslims is still very important. Women and girl support groups are very important adaptive social structures that function as social support to community members.

In all the discussion groups there was constant reference to these structures as the possible avenues or consultative groups with regard to initiation of activities of whatever nature. The groups are gender as well as age specific thus giving a potential programme the specificity and flexibility in the design of a variety of approaches.

Given the sensitivity, within which EC is initially regarded, its introduction should be within the broad framework of Reproductive Health (RH) services, which are already embraced by the community. Reproductive health services have already been spear headed by other organizations notably NCCK besides IRC. This means that EC could be provided through the various service delivery levels (for example CHWs and the health facilities) within the camp to increase the level of accessibility and coverage. Although IRC and NCCK are providing education on RH topics in Kakuma, preliminary survey results indicate that provision of education needs to be intensified. Thus to facilitate the use of EC, an initial step would be to train the relevant cadre of people that would be providing EC and dissemination of general health information at the same time. 


\section{Recommendations}

The objectives of conducting the baseline qualitative and quantitative research were to assist in:

- Suggesting specific revisions in the training curricula for the health care providers

- Development and mode of dissemination of IEC messages in the community and health facilities.

Results generated from the research highlighted the lack of correct information regarding content, number of and timing when the pills need to be taken among all levels of heath care providers. However, what was most encouraging was the fact that, by and large, the heath care providers were every supportive for provision of ECs and willing to provide this method at health facilities and in the community.

However, regarding dissemination of information and hence provision of ECs at community level, there was a consensus, by and large, that gender violence including rape is a major reproductive health concern in Kakuma and hence participants in all discussion groups acknowledged the role that ECs can play in reducing post-rape pregnancy rate. However, their concern, especially among the community elders, were the mode of dissemination of ECs as they strongly advocated this should be through the active participation and support of community elders. The avenues for dissemination of information recommended included health facilities, youth groups, women support groups, drama and puppetry sessions among others. 
APPENDIX 1

Emergency Contraception Project-Kakuma

IN-DEPTH INTERVIEWS WITH SERVICE/PROVIDERS IN KAKUMA REFUGEE CAMP

\section{Interviewer's guide}

Provider name:

Provider sex (circle one): Female Male

Home Country

Name of facility:

Location of facility:

Interview date:

Interview start time:

Interview end time:

Interviewer's initials:

\section{Introduction.}

Thank you for seeing me. I am here today because we are planning a pilot project to consider introducing emergency contraception more widely into Refugee Settings. We are working with IRC, LWF, NCCK and MOH, and we are interviewing health providers as well as policy makers, Youth and women in the reproductive age group.

In order to help us plan the project and to evaluate it later on, we would like to ask you some questions. Please try to answer the questions honestly. Do not worry if you are not sure about the answer. The interview should not last more than 30 minutes. We will not report your name in any way when we write up the final results. We wish only to collect it for our records. All the individual information we collect will be kept confidential, and only information that cannot be linked back to you will be shared outside the research project.

Are you willing to participate in our project $\quad$ Yes No 


\section{Demographic information.}

Before we begin, can we get some background information?

1. When were you born?

2. What is your job here? (Circle one).

Doctor
Nurse
Clinical Officer
Nurse Aide
CHW
CBD

Other:

3. How many years of experience do you have in providing health services:

4. How long have you been working in Kakuma:

5. Have you had any specialized training in family planning? (Circle one). Yes No If yes, please specify: 


\section{Description of facility and services currently offered.}

Let us begin by talking a little bit about the health services your facility offers now.

6. Which family planning methods do you offer? In each case, can you tell us whether you offer information or also supplies? Please try to estimate how many women receive each method in an average month. (Complete grid below.)

\begin{tabular}{|l|l|l||}
\hline Method & $\begin{array}{l}\text { Number of clients } \\
\text { offered information } \\
\text { on the method (in an } \\
\text { average month)? }\end{array}$ & $\begin{array}{l}\text { Number of clients provided } \\
\text { with supplies (in an average } \\
\text { month)? }\end{array}$ \\
\hline IUD & & \\
\hline Combined Pills & & \\
\hline Mini Pills & & \\
\hline Condoms & & \\
\hline Diaphragms/Caps & & \\
\hline Vasectomy & & \\
\hline Tubal ligation & & \\
\hline $\begin{array}{l}\text { Emergency } \\
\text { contraception }\end{array}$ & & \\
\hline Other: & & \\
\hline
\end{tabular}

7. Where do you obtain your contraceptive supplies? (Record exact response).

8. Which brands of oral contraceptives do you normally stock? (Record exact response).

9. Which of these brands do you have in stock right now? (Circle brands in stock from brands listed above in question 6). 
10. Do you encounter any difficulties keeping pills in stock? (Record exact response).

11. What other reproductive health services do you offer? (Ask each category and circle one response).

$\begin{array}{llll}\text { Diagnostic: } & \begin{array}{l}\text { Pregnancy testing? } \\ \text { STD screening? }\end{array} & \begin{array}{l}\text { Yes } \\ \text { Yes }\end{array} & \text { No } \\ & & & \\ \text { Curative: } & \text { STD treatment? } & \text { Yes } & \text { No } \\ & \text { Evacuation? } & \text { Yes } & \text { No }\end{array}$

12. How would you describe your typical client/patient population? (Ask each category and circle one response).

$\begin{array}{clll}\text { Average age: } & \text { Young } & \text { Medium } & \text { Older } \\ \text { Age range: } & \text { Varies a lot } & \text { Varies somewhat } & \text { Does not vary much } \\ \text { Ethnicity } & \text { Sudanese } & \text { Somali } & \text { Other }\end{array}$

13. How many clients or patients does the facility generally see each week for all services combined?

14. What days and times is this facility open? (Note times for each day the clinic is open).

Monday:

Tuesday:

Wednesday:

Thursday:

Friday:

Saturday:

Sunday:

\section{Definition of emergency contraception.}

Now let's talk about emergency contraception. Some people have heard of it. Some people have not heard of it.

15. First of all, have you heard of it? (Circle one). Yes No

If answer is "no," skip question 16 \& 17(explanation). 
16. People have different ideas about what emergency contraception is. Can you tell me what impression comes to your mind when we think of this term? (Record exact response).

17. Can you tell me when you first heard of it and where? (Record exact response).

When:

Where:

Just to be sure we are all working from the same idea, I'd now like to give a short summary of the method. In case you weren't sure, emergency contraception is a term used to describe methods of contraception that women can use after sex to help prevent pregnancy. For example, regular oral contraceptives used in a higher dose and taken within a few days after unprotected sex work well as emergency contraceptives. Sometimes this therapy is called the morning after pill. Emergency contraceptive pills must be started within 72 hours after unprotected sex and they are about $75 \%$ effective. The ingredient is the same as the regular birth control pill, but a larger dose. Some women who take it experience relatively minor side effects, such as nausea and vomiting. Although it is possible to get emergency contraception in Kenya, it has not yet widely known. As you can see from the name, the method is for contraceptive emergencies and not for use as a regular method.

18. Do you have any questions about this method? (Note the questions and answer them).

\section{Current provision of emergency contraception.}

19. Do you offer emergency contraception? (Circle one). Yes No

If answer is "no," skip to question 32.

20. When did you start offering emergency contraceptives?

21. Why did you start to offer emergency contraceptives? (Record exact response). 
22. How often do you give emergency contraception on average in a month?

23. Can you describe the typical emergency contraceptive clients? (Record response).

24. Do you offer emergency contraception to some clients but not to others? What are the selection criteria you use? (Record response).

25. Do you see any variation in the need for EC at different times during the year? Please describe. (Record comments).

26. Which regimens do you offer? (Record exact protocol and brands used.)

27. What if anything is the typical counselling you offer on emergency contraception?

(Record comment.)

28. Do you have any client materials on emergency contraception? (Circle one). Yes No (If "yes, " request a copy).

29. Do you have any written guidelines that you use for emergency contraception? (Circle one). Yes No. (If "yes," request a copy). 
30. Do you charge for emergency contraception? (Circle one). Yes No If yes, how much? KSh

31. What problems have you encountered with offering this service? (Record comment).

(Skip the next question.)

32. If you do not offer emergency contraception, why not? (Record comment).

\section{Perceptions of emergency contraception.}

Now let's talk about emergency contraception very generally.

33. What do you think about this method? (Record comments).

34. Do you think it is/could be a useful method for your facility? Why or why not? (Record comments).

35. What are the advantages you see? (Record comments). 
36. What are the limitations? (Record comments).

37. What about for Kenya generally? Do you think it could be a useful method? Why or why not? (Record comments).

38. If the method became more widely available in Kenya, who do you think should be able to offer it? (Probe: Only physicians? Nurses? CBD workers? Pharmacists? Clinical officers? Other?) Through what channels should it be made available? (Record comments.)

and who should be offered this method?

39. What would be the best ways to inform women about emergency contraception? (Record comments).

\section{Advance distribution.}

In some other countries such as Scotland and the United States, providers are giving packets of pills to women in advance. The women can take them home and use them as emergency contraception in case they need them without having to come back to the clinic. 
40. What do you think of this idea for Kenya? (Record comments).

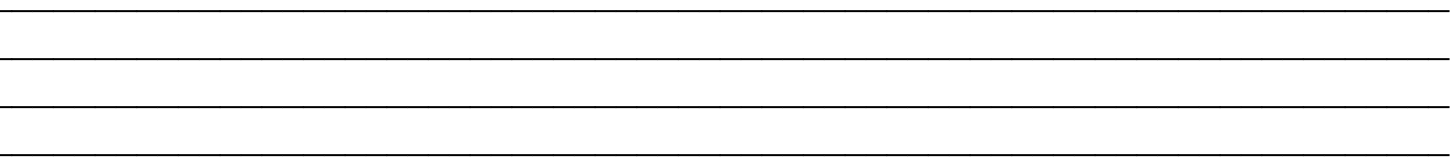

41. Would this be a suitable idea in this facility? (Record comments).

42. Which women would be suitable to receive advance supplies? (Record comments).

\section{Future plans for emergency contraception.}

43. Would you like to see emergency contraception become more easily available at your site? (Record comments).

44. What do you see as the main challenges in offering emergency contraception here at your site? (Record comments).

45. Do you have any ideas to share with us for overcoming these challenges as we plan our pilot project? (Record comments). 
46. What about in Kenya generally? Would you like to see emergency contraception become more easily available? (Record comments).

47. Some people are in the process of developing a special package that would contain the right dose of oral contraceptives and the right directions for use as emergency contraception. Even though the pills are the same, a pilot test will see if providers prefer the special package. Do you think such a special package would be useful? (Record comments).

48. What do you think are the main ways we could tell if such a pilot project has been a success? (Record comments).

\section{Conclusion.}

We have now reached the end of the prepared questions I have for you.

49. Is there anything else you would like to add? (Record any comments).

50. Would you like to ask me any questions? (Note questions and answer them).

Thank you very much for your time. We will contact you in the near future to share the findings and discuss how to move forward in trying to make this method readily available for settings such as this.

(Note ending time of interview: . Also mark ending time on front page). 
APPENDIX 2

Emergency Contraception Project - Kakuma

\section{SURVEY AMONG REFUGEE WOMEN IN THE KAKUMA CAMPS IN KENYA}

Date of interview:

Camp

Phase/zone

Home Country

Start time of interview:

End time:

Client number: (Number consecutively, 1 to 100 at each site).

Interviewer initials:

\section{Introduction.}

We are planning a pilot project to introduce emergency contraception more widely into the camps. As part of the planning for this project, we are talking with women in Kakuma. Would you be willing to answer some general questions? The survey will take less than 15 minutes. We will not collect your name, and the information will not become part of your record any where. You are free to refuse if you prefer, since doing so will not affect your services here in any way. We also promise to keep all information we collect confidential. Are you willing to participate?

\section{Background information.}

The first questions are for background.

1. How old are you?

2. How many years of education have you completed?

3. Have you ever been pregnant? (Circle one). Yes No

4. Do you use any method of contraception? (Circle one). Yes No 
5. If you use a method of contraception, which one is it? (Circle one).
a) pills
f) withdrawal
b) condoms
g) rhythm
c) IUD
h) other:
d) female sterilization
e) male sterilization

\section{Knowledge about emergency contraception.}

And now let us discuss the main subject of our survey.

6. If a woman has had unprotected sex, for example if she has been raped, or her condom broke, or she forgot to use a method, is there anything she can do if she does not wish to become pregnant? (Do not read choices. Get unprompted response. Circle the option closest to woman's answer. Unless the answer is very close, record the woman's exact answer on the line marked "other:").
a) there is nothing she can do
b) She can go to the hospital or clinic (what for:
c) she can use some traditional remedies (specify:
d) she must wait to see if she will become pregnant and then she can get an abortion
e) she can use emergency contraception
f) other:
g) not sure

The next questions are about a medication called emergency contraception. Emergency contraception is also sometimes called the morning after pill. Some people have heard of it. Some people have never heard of it.

7. Have you ever heard about emergency contraception? (Circle one). Yes No If the client answered "no," skip to next section entitled "Attitudes."

8. When did you first hear of emergency contraception? 
9. Where did you first hear about emergency contraception? (Do not read choices. Circle all that she mentions if she mentions more than one. Unless the woman's choices are very close, record her exact answer on the line marked "other:").
a) clinic
b) friends, family (word of mouth)
c) read about it in news, women's magazines
d) other:
e) not sure

10. Where do you think a woman can get emergency contraception? (Do not read choices out loud. Circle all that are mentioned.)
a) hospital/camp clinic
d) pharmacy
b) community health worker
c) private clinic
e) shop
f)

other:

11. For it to be successful, how should emergency contraception be used? (Record exact answer).

12. Will emergency contraception work if a woman notices that her period is late? (Circle one). Yes No Not sure

13. How well do you think emergency contraception works if used correctly? (Circle one).
a) almost $100 \%$
b) $75 \%$
c) $50 \%$
d) $25 \%$
e) not sure 
14. How soon after unprotected sex must emergency contraception be used in order for it to work? (Circle one).
a) immediately afterwards
b) within 24 hours
c) within 72 hours
d) within one week
e) anytime before the missed period
f) not sure

15. Do you think the emergency contraception pill is: (Circle one).
a) the same as the regular birth control pill
b) the same but stronger
c) completely different
d) not sure

\section{Attitudes toward emergency contraception}

In case you weren't sure, emergency contraception is a term used to describe methods of contraception that women can use after sex to help prevent pregnancy. For example, birth control pills used in a higher dose and taken within a few days after unprotected sex work well as emergency contraceptives. Sometimes this therapy is called the morning after pill.

Emergency contraceptive pills must be started within 72 hours after unprotected sex and they are about $75 \%$ effective. The ingredient is the same as the regular birth control pill, but a larger dose. Some women who take it experience relatively minor side effects, such as nausea and vomiting. Although it is possible to get emergency contraception in Kenya, it is not yet widely known. As you can see from the name, the method is for contraceptive emergencies and not for use as a regular method.

16. What do you think about this method? (Record exact answer).

17. Would you have any concerns about this method? (Circle one). Yes No If she answers "no," skip to question 19. 
18. If you have concerns, what would they be? (Do not read choices. Circle all that are mentioned. Unless the woman's choice is very close, record her exact words on the line marked "other:").
a) it is not yet familiar to me
b) it might cause health problems
c) it will harm the baby if it does not work
d) women might not be able to have babies properly later
e) it is abortion
f) it might not be legal
g) it will cause women to get more STDs, and possibly HIV
h) if men learn it is available, they will pressure women to use it
i) it will be abused by women who take it too often instead of a regular method j) other:

19. Do you disagree or agree with the following statement: Emergency contraception is suitable for women in a refugee status. (Circle one).
a) strongly agree
b) agree
c) neutral
d) disagree
e) strongly disagree

20. Where do you think the method should be available? (Ask each choice in turn and circle yes or no for each one).
a) from a doctor: Yes No
b) from any staff at a health facility: Yes No
c) from the pharmacy: Yes No
d) from the community health worker or CBD: Yes No
e) from any shops where medicines are sold: Yes No

21. Do you think there are any groups of women who should not get emergency contraception? (Do not read choices. Collect unprompted response. Circle all that are mentioned. When she has mentioned a choice, ask for further choices by saying "Anyone else?" until she has no more responses).
a) no restrictions, it should be available for anyone who needs it
b) it should be available for anyone except not for young girls
c) only for married women 
d) only for women who have been raped

e) only if regular contraception fails

f) other:

22. From what you know about emergency contraception, do you think you would ever use it or recommend it to a friend if the need arose? (Circle one).
a) yes
b) no
c) not sure

23. Do you think men would object to their women using Emergency Contraceptive pills?
a) no, they would not
b) yes, they would
c) not sure

24. What are the best ways to inform women about emergency contraception? (Record exact response.)

\section{Conclusion}

Thank you very much for your time. Before we conclude, would you like to ask me any questions about emergency contraception? (Note and answer all questions.) Thank you again.

Questions asked by client:

(Note end time of interview: . Also mark time on front page.) 


\section{APPENDIX 3:}

\section{Emergency Contraception Project}

FOCUS GROUP DISCUSSIONS WITH YOUTH,MEN,AND WOMEN AT KAKUMA REFUGEE CAMP, KENYA

Study information.

Survey population: Two focus groups (one male and one female) of youth in each of the 3 camp locations (Kakuma I, Kakuma II and Kakuma III). Each of the six focus groups should have between six and ten participants. Youth must be recruited without revealing the topic of the discussion (they may be told that the topic is about "health" but anything more detailed may bias the results.) Compensation $=$ KSh 20 per participant. Each interview will take about 1 hour.

Timeline: All interviews must be complete by November30,1999 Tapes should be transcribed by January 2000 .

Data collection: Each group should be taped and transcribed. 


\section{Duties of focus group researchers:}

1) Recruit youth

2) Moderate group (moderator)

3) Operate tape recorder and take extensive notes (note taker)

4) Transcribe tapes

\section{Organizing and recruiting groups}

a) There should be 6 to 10 people per group (recruit up to 12, as not all will show up). Four groups in each camp (one men, one women and one youth boys and girls separate). Give each participant you recruit written directions with the date, time and place of the group.

b) Do not state the topic of the focus group in advance. Doing so will bias the study by influencing the type of people who are likely to accept. If you must say something, state that the group will discuss a current issue in health.

c) Organize a space that will have no interruptions, and that is quite and private.

\section{Leading the focus group (moderator)}

a) Seat the participants near one another so that they can talk together. Inform them that you are running a focus group about emergency contraception. Tell them that the study is being conducted by the Population Council and IRC, with the permission of the UNHCR. They can obtain a copy of the study when it is ready fromIRC

b) State that you are just interested in opinions. There will be no discussion about personal behavior.

c) Explain that if anyone does not wish to participate, she or he may leave. Anyone can leave the group at any point in the discussion if they feel uncomfortable.

g) While moderating the group, do not correct wrong notions. If they ask, tell them that at the end, there will be a chance for them to ask questions of you if they want. You are a facilitator, not an instructor.

h) Ask if they have any questions before you turn on the tape recorder. When any questions have been answered, turn on the tape recorder and start the discussion.

i) During the discussion, summarize every fifteen minutes or so. This process will help later with analysis and will also give participants a chance to clarify or add anything they forgot to mention. The summary also lets them know you are listening carefully to them.

j) At the end of the group, thank the participants, and answer any questions they have. If the participants wish, you can correct misperceptions you heard. 


\section{Taking notes (note taker)}

a) Draw a brief diagram of the layout of the group, noting the position of the moderator and note taker. Note peoples initials as they say them on this diagram, and then use initials only while taking notes during the group. Note the date, place, and starting and ending time of the group.

b) Sit unobtrusively back from the main group, but in a place from which you can clearly see who is saying what.

c) Bring plenty of copies of the demographic data questionnaire and background impressions worksheet and plenty of pens and pencils. Help to hand these out when the moderator indicates.

d) Clearly label the tapes with the date, place and time of the focus group before starting.

e) Use two tape recorders at each group. You need at least one to be going in case you need to turn over the tapes, and it often happens that one tape recorder breaks or gets stuck. Check each tape recorder to make sure it works and that the batteries are new. Start the tape recorder when the moderator instructs you to.

f) Take notes of each comment made listing the initial of the person making the comment. Include notes on the questions or comments posed by the moderator. Try to catch distinctive phrases word for word. Otherwise, catch the sense of each comment.

g) Watch and note non-verbal responses, such as when people nod, when everyone seems to agree with a point, and when people laugh.

h) At the end of the group, dispense the compensation.

\section{Transcribing tapes}

a) Transcribe each tape exactly as the words are spoken on it. Use a copy of the notes from the note taker to help you. 


\section{Moderator's guide.}

\section{Introduction.}

Thank you for coming. I am Esther Muia, from the Population Council. With me is Joyce Olenja from Nairobi. The purpose of today's discussion is to learn what you think about a certain method of contraception. We are planning a pilot project to introduce this method more widely, and your views will help us plan a better project. We are doing this study with youth at Kakuma, as well as with many members of the medical profession and some officials in health policy.

In the discussion today, we will not ask you anything about your own behaviour, only about your opinions. We are not recording your name, and nothing you say in this group will be linked back to you in any way. We would like to record this session in order to help us with our analysis, and we will keep it entirely confidential. Do you mind if we tape the discussion?

Please try to speak frankly. Do not worry if you are not sure about the answer. Although we will not correct any mistaken impressions during the group, we will tell you at the end any information you want to know, and we will leave a time for you to ask us questions then. It is important for us to hear from everyone, so even if you are normally shy, try to speak up. If you are normally talkative, try to state your views briefly to give time to the shy ones.

Periodically during the focus group, I will stop and summarize what I think you have said. This is a time for you to add anything you did not get a chance to say and also a time for you to correct me if I have misunderstood something.

The discussion will last about one hour and we will have a soda as we go on . Are you willing to participate in our project? Note that you may leave the group at any time if you change your mind. (Anyone who is not willing may leave).

\section{Demographic information and background impressions of emergency contraception.}

Before we begin, we would like to get some brief background information. (Hand out brief questionnaire and pencils to everyone.)

When you are finished filling in the top part of the questionnaire, please write down anything you may have heard or you may think about emergency contraception. Note that this method is also sometimes called "morning after pills." Let us know if you have heard of either of these names before. Don't worry if what you write is wrong or right, and don't worry if you have never heard of this method. Just write down your impressions.

(Give them a few minutes to complete the paragraph and collect the sheets.)

Now, do you have any questions before I turn on the tape recorder?

(Turn on tape recorder). 


\section{Possibilities for avoiding pregnancy generally.}

Let us begin by talking about contraception. Do you know of any ways for women to avoid having babies? What can she do? What do you think about these things?

\section{Possibilities for avoiding pregnancy after unprotected sex.}

Now let's talk about a contraceptive emergency for women. If a woman has had sex, and she was using a condom, but the condom burst, is there anything she can do? Similarly, if a woman was raped, or tricked into having sex with a man, can she do something to avoid pregnancy?

(Summarize).

\section{Initial impressions of emergency contraception}

Now I would like to share what you have written about emergency contraception. Again, don't be shy and don't worry if you do not have the right information. This exercise is really just to get the ball rolling.

(Hand out the background sheets randomly and ask them to read out loud the one they have been given. If nobody has heard of emergency contraception or if nobody has information that is even vaguely correct, skip to the section with the definition and start there.)

\section{Knowledge about emergency contraception.}

(Ask the following types of questions during the discussion as the opportunity arises:)

How a person might hear of emergency contraception? (Probe: friends, news, clinics?)

Where could you get more information?

Where could a woman get the method?

Can you think of circumstances when it would be appropriate to use emergency contraception? And are there times when it would not be appropriate?

Does it always work? Are there times of the month when it works best?

Does it have side effects? If so, what type and are they serious? Would the side effects prevent you from taking this or recommending it to a friend?

Have you heard about its use for rape victims? Do you think it is a good idea? 
Do you know what the effective ingredient is? Is it similar to anything else you are familiar with?

(Summarize. Ask"Have I understood you correctly?).

\section{Brief definition of emergency contraception.}

Just to make sure we are all talking about the same thing, let me review very briefly what the method is. In case you weren't sure, emergency contraception is a term used to describe methods of contraception that women can use after sex to help prevent pregnancy. For example, birth control pills used in a higher dose and taken within a few days after unprotected sex work well as emergency contraceptives. Sometimes this therapy is called the morning after pill. Emergency contraceptive pills must be started within 72 hours after unprotected sex and they are about $75 \%$ effective. The ingredient is the same as the regular birth control pill, but a larger dose. Some women who take it experience relatively minor side effects, such as nausea and vomiting. Emergency contraception is used widely overseas, for example in England and in the United States. Although it is possible to get emergency contraception in Kenya and other African countries, it is not yet widely known. As you can see from the name, the method is for contraceptive emergencies and not for use as a regular method.

\section{Attitudes.}

Now, what do you think about emergency contraception?

(Ask the following types of questions as the opportunity arises).

Do you think this method should be available in the camp?

Should it be available to youth?

Is there any reason why you think it should be available? Is there any reason why you think it should not be available?

Is there something about this method that makes you uncomfortable?

What about when a couple does not use contraception or when a method fails, for example when a condom breaks? Is emergency contraception a good idea?

What about in cases of rape?

Would you recommend emergency contraception to a friend in case of need?

(Summarize). 


\section{Ideal availability and access.}

The last topic I would like to talk about is ideal availability and access.

Should more people be informed about emergency contraception?

Who needs this information the most? How can we inform such people?

Do you think emergency contraception is already available easily enough? How would you find it if you needed it?

Where would be the best place to make it available?

Do you think more people would use it if they knew it was available?

In some countries, women can be given a packet of emergency contraception in advance. That way she can keep in with her in case she needs it. Do you think this idea would be suitable in camp? Why or why not?

(Summarize).

\section{Conclusion.}

Now we are reaching the end of our discussion. Is there anything else you wish to add? Is there anything you would like to ask me? In case you prefer to ask anonymously, just write them on these sheets and I will answer them. You may also write any comments you may have. (Hand out sheets of paper and pencils and let them submit any questions in writing. Collect and answer the questions. Save the questions for later analysis.)

Thank you very much for your participation. (Dispense compensation.) 


\section{Focus group on emergency contraception: Background information}

Date:

Initials:

Site (circle one): Kakuma I Kakuma II Kakuma III

Sex (circle one): $\quad$ Female Male

Year of birth:

Country:

Level of school (circle one): Primary Secondary College None

Tribe:

Please write a brief paragraph here about your impressions of emergency contraception. Emergency contraception is also sometimes called the morning-after pill. Have you heard of either of these terms? Where? Do you have any ideas what they could be? 